\section{1} W.).

\title{
mTOR-neuropeptide Y signaling sensitizes nociceptors to drive
}

\section{neuropathic pain}

Lunhao Chen ${ }^{1 \dagger}$, Yaling $\mathrm{Hu}^{2,3,4 \dagger}$, Siyuan $\mathrm{Wang}^{1 \dagger}$, Kelei Cao ${ }^{2,3,4}$, Weihao Mai ${ }^{2,3}$, Weilin Sha ${ }^{5}$, Huan $\mathrm{Ma}^{2,3,4}$, Yong-Jing Gao ${ }^{5}$, Shumin Duan ${ }^{2,3,4}$, Yue Wang ${ }^{1 *}$, Zhihua Gao ${ }^{2,3,4 *}$

${ }^{1}$ Spine Lab, Department of Orthopedic Surgery, The First Affiliated Hospital, Zhejiang University School of Medicine, Hangzhou 310003, China

${ }^{2}$ Department of Neurobiology and Department of Neurology of Second Affiliated Hospital, NHC and CAMS Key Laboratory of Medical Neurobiology, Zhejiang University School of Medicine, Hangzhou 310058, China

${ }^{3}$ The MOE Frontier Research Center of Brain \& Brain-machine Integration, Zhejiang University School of Brain Science and Brain Medicine, Hangzhou 310058, China

${ }^{4}$ Liangzhu Laboratory, Zhejiang University Medical Center, 1369 West Wenyi Road, Hangzhou 311121, China

${ }^{5}$ Institute of Pain Medicine and Special Environmental Medicine, Nantong University, Nantong 226019, China

$\dagger$ These authors contributed equally to this work.

* Correspondence: zhihuagao@zju.edu.cn (Z.G.) and wangyuespine@zju.edu.cn (Y. 


\section{Abstract}

Neuropathic pain is a refractory condition that involves de novo protein synthesis in the nociceptive pathway. The mechanistic target of rapamycin (mTOR) is a master regulator of protein translation; however, mechanisms underlying its role in neuropathic pain remain elusive. Using spared nerve injury-induced neuropathic pain model, we found that mTOR is preferentially activated in large-diameter dorsal root ganglion (DRG) neurons and spinal microglia. However, selective ablation of mTOR in DRG neurons, rather than microglia, alleviated neuropathic pain. We show that injuryinduced mTOR activation promoted transcriptional induction of NPY likely via signal transducer and activator of transcription 3 (STAT3) phosphorylation. NPY further acted primarily on Y2 receptors (Y2R) to enhance nociceptor excitability. Peripheral replenishment of NPY reversed pain alleviation upon mTOR removal, whereas Y2R antagonists prevented pain restoration. Our findings reveal an unexpected link between mTOR and NPY in promoting nociceptor sensitization and neuropathic pain, through NPY/Y2R signaling-mediated intra-ganglionic transmission.

Keywords: neuropathic pain; mTOR; NPY; STAT3; Y2 receptor; nociceptor; mechanoreceptor 


\section{INTRODUCTION}

Chronic pain, the leading cause of long-term human disability, poses a heavy health burden to the society. Nerve injury-induced neuropathic pain accounts for approximately one fifth of the chronic pain population (van Hecke et al., 2014). It is characterized by persistent hyperalgesia, allodynia and spontaneous pain. Long-lasting sensitization of the nociceptive pathway, leading to a reduced pain threshold, has been considered a major mechanism mediating the persistent hypersensitivity in neuropathic pain (Costigan et al., 2009).

Accumulating evidence has shown that nerve injury-induced de novo gene expression contributes to the maladaptive responses in both the peripheral and central nociceptive circuits, thereby promoting nociceptive sensitization and pain hypersensitivity (Costigan et al., 2009; Melemedjian and Khoutorsky, 2015). Elevation of G proteincoupled receptors (GPCRs), such as GPR151, coupled with ion channels in the injured dorsal root ganglion (DRG), has been shown to facilitate the generation of ectopic action potential in nociceptive neurons and promotes pain (Geppetti et al., 2015; Xia et al., 2021).

Other than ion channels and GPCRs, prominent induction of neuropeptides, including neuropeptide Y (NPY), galanin (Gal), neurotensin (NTS) and cholecystokinin (CCK), have also been observed in DRG neurons after nerve injury (Reinhold et al., 2015; Wu et al., 2016; Xiao et al., 2002). The 36-amino acid peptide, NPY, is one of the most robustly upregulated neuropeptides in DRG neurons after nerve injury (Wakisaka et al., 
1991). However, mechanisms underlying its induction remain unknown. Moreover, conditional knockdown of spinal cord NPY increased tactile and thermal hypersensitivity primarily through $\mathrm{Y} 1$ receptor (Y1R) in nerve injury-induced neuropathic pain models (Solway et al., 2011; Nelson and Taylor, 2021), whereas subcutaneous injection of NPY or Y2 receptor (Y2R) agonist exacerbated pain after nerve injury, suggesting a biphasic role of NPY in neuropathic pain at different sites (Sapunar et al., 2011; Tracey et al., 1995; Arcourt et al., 2017). It remains to be elucidated how NPY was induced after injury and whether NPY plays opposing roles through different receptors in the nociceptive pathway.

The mechanistic target of rapamycin (mTOR), a master regulator of protein translation, plays a pivotal role in regulating cell growth and metabolism. Deregulation of mTOR signaling has been linked to various human diseases, including cancer, obesity and neurodegeneration (Saxton and Sabatini, 2017; Carlin et al., 2018; Laplante and Sabatini, 2013). Activation of mTOR has been observed in the DRG and spinal cord in neuropathic pain models, as well as morphine-induced chronic pain (Abe et al., 2010; Zhang et al., 2013; Xu et al., 2014; Melemedjian et al., 2011; Price and Géranton, 2009). Pharmacologic blockade of mTOR activity has been demonstrated to reduce pain (Geranton et al., 2009; Asante et al., 2010; Obara et al., 2011; Tateda et al., 2017; Norsted Gregory et al., 2010; Xu et al., 2011). However, several studies found that inhibiting mTOR complex 1 (mTORC1) resulted in unexpected mechanical allodynia, through an insulin receptor substrate-1 (IRS-1)-dependent negative feedback activation of extracellular signal-regulated kinase (ERK) in primary sensory neurons 
80 (Melemedjian et al., 2013; Melemedjian and Khoutorsky, 2015), leaving the role of

81 mTOR and underlying mechanisms in pain regulation to be further clarified.

82 Combining genetic manipulation, transcriptomic profiling with electrophysiological

83 recording, we uncover a previously unrecognized link between nerve injury-triggered

84 mTOR activation and NPY induction in DRG neurons. We further demonstrate that

85 mTOR-mediated NPY production enhances nociceptor excitability and promotes pain

86 hypersensitivity through Y2R in DRG. Although mTOR-related signaling has been

87 extensively studied, we present the first evidence for mTOR-regulated NPY signaling

88 in driving neuropathic pain development. 


\section{RESULTS}

\section{Nerve injury induces mTOR activation in subsets of DRG neurons and spinal cord microglia}

To examine the status of mTOR activation after nerve injury, we carried out western blot analysis of L4 and L5 DRG and spinal dorsal horn (SDH) tissues from mice at different time points after the spared nerve injury (SNI) surgery (Figure 1A). The activity of mTOR was assessed by the levels of phosphorylated S6 protein (p-S6), a key and downstream effector of mTOR. As shown in Figure 1B and C, p-S6 was substantially upregulated in the ipsilateral DRG one day after the nerve injury and lasted for at least 7 days $(p<0.05)$. These data are consistent with elevated mTOR activity in DRGs after peripheral nerve injury (Abe et al., 2010).

To further determine the identity of cells with mTOR activation, we performed immunofluorescence analysis using anti-p-S6 antibody along with different markers. Size frequency analysis showed that expression of p-S6 in DRG neurons was mainly in medium and large sizes at contralateral and ipsilateral DRGs after SNI (Figure 1-figure supplement 1A). In the contralateral DRG, positive p-S6 labeling, reflecting basal mTOR activity, was observed in a small subset of $\mathrm{CGRP}^{+}$peptidergic neurons (9.7\%) but a large fraction of $\mathrm{NF} 160 / 200^{+}$neurons, reminiscent of large-sized A-fiber mechanoreceptors (43.7\%). In the ipsilateral DRG, a substantial increase of p-S6 ${ }^{+}$cells in NF160/200 ${ }^{+}$large-sized mechanoreceptors (from $43.7 \%$ to $71.2 \%, p<0.01$ ) and $\mathrm{CGRP}^{+}$peptidergic neurons (from $9.7 \%$ to $18.7 \%, p<0.05$ ) was observed 3 days after 
SNI (Figure 1D-F). Notably, no elevation of mTOR activity was observed in IB4 ${ }^{+}$non-

By contrast, western blot analysis of p-S6 from the SDH tissue extracts detected no differences between the contralateral and ipsilateral spinal cords following SNI $(p>0.05$,

Figure 1H and I). Given that western blot analysis detects the gross mTOR activity in the SDH, which may mask changes in sparsely distributed cells in the spinal cord, we carried out dual-labeling of p-S6 with different cellular markers, including NeuN (neurons), GFAP (astrocytes) and Iba1 (microglia). No changes were observed in p-S6 ${ }^{+}$ neurons or astrocytes between the contralateral and ipsilateral SDH within 1 week following the injury (Figure 1-figure supplement 1B-D). However, the number of p$\mathrm{S}^{+}$microglia $\left(\mathrm{GFP}^{+}\right)$in the superficial layers of ipsilateral SDH was robustly increased

121 from day 3 to 7 post SNI in $C x 3 c r 1^{E G F P /+}$ mice ( $p<0.05$, Figure $\mathbf{1 J}$ and $\left.\mathbf{K}\right)$. Together, 122 our results demonstrate that peripheral nerve injury induces mTOR activation mainly in large-sized DRG mechanoreceptors and SDH microglia.

\section{Blocking mTOR activity delays pain development}

To further determine the contribution of mTOR signaling in neuropathic pain, we administered rapamycin, an mTORC1 inhibitor, by intraperitoneal injection to systematically blocking the mTORC1 activity. Meanwhile, BrdU was injected into the mice to label proliferating microglia (Figure 2A). Daily intraperitoneal administration of rapamycin from one day before to 7 days after the SNI significantly inhibited mTOR 
Using von Frey tests, we found that systemic rapamycin administration delayed the appearance of mechanical allodynia for 5 days after the nerve injury $(p<0.05$, Figure 2B). Rapamycin treatment also reduced the total number of microglia (Vehicle: 757.7 \pm 15.4 per $\mathrm{mm}^{2}$, Rapamycin: $463.1 \pm 20.7$ per $\mathrm{mm}^{2}, p<0.001$ ) (Figure $2 \mathrm{C}$ and $\mathbf{D}$ ) and the percentage of proliferative microglia $\left(\mathrm{BrdU}^{+} \mathrm{Iba}^{+}\right.$) (Vehicle: $86.9 \% \pm 1.1 \%$, Rapamycin: $73.1 \% \pm 2.1 \%$ ) in the superficial layers of ipsilateral SDH at day 7 after SNI (Figure 2C and E). These data demonstrated that blocking mTOR signaling delayed pain and suppressed nerve damage-induced microgliosis.

\section{Selective ablation of mTOR in DRG neurons but not in microglia alleviates neuropathic pain}

To further discern the contributions of neuronal or microglial mTOR in neuropathic pain, we crossed specific Cre mouse lines (Adv $v^{\text {cre }}$ or $\left.C x 3 c r 1^{\text {creER }}\right)$ with $M t o r^{f l f l}$ mice to selectively delete Mtor gene in primary sensory neurons or microglia, respectively. We observed complete elimination of p-S6 in DRG neurons and unchanged p-S6 levels in SDH in $A d v^{c r e}:: M t o r r^{f l / f l}$ (Mtor-cKO ${ }^{A d v}$ ) mice 7 days after SNI (Figure 3A and B), demonstrating the selective removal of Mtor in primary sensory neurons. Examination of sensory perception and motor activities found no significant differences between the control and Mtor-cKO ${ }^{A d v}$ mice at basal states (Figure 3-figure supplement 1). However, Mtor-cKO ${ }^{A d v}$ mice exhibited delayed development of mechanical allodynia (Figure 3C) and cold allodynia (Figure 3E) than the controls after SNI, as well as alleviated heat hyperalgesia (Figure 3D). Moreover, Mtor-cKO ${ }^{A d v}$ mice had lower 
152 difference scores in response to mechanical stimulation than the Mtor ${ }^{\text {fl/fl }}$ mice in a two-

153 chamber CPA assay that assesses the aversive responses to pain, suggesting that mTOR

154 deletion in DRG neurons alleviated aversive responses to noxious stimuli (Figure 3F).

155 To further examine whether microglial mTOR activation also contributes to

156 neuropathic pain, we selectively deleted Mtor in microglia by injecting tamoxifen into

157 the $C x 3 c r 1^{c r e E R /+}:: M t o r{ }^{f l / f l}$ mice (Mtor-cKO ${ }^{M G}$ mice) 4-6 weeks before the SNI surgery

158 (Figure 4A and Figure 4-figure supplement 1A) (Gu et al., 2016). Cre-mediated

159 recombination of Mtor gene in the central nervous system (brain and spinal cord) was

160 detected by PCR analysis (Figure 4-figure supplement 1B) and ablation of mTOR in

161 microglia was verified by immunofluorescence analysis (Figure 4B). At day 7 post SNI,

162 we observed a reduction in the number of microglia (Figure 4C and D) and the

163 percentage of mitotic microglia $\left(\mathrm{BrdU}^{+} \mathrm{Iba}^{+}\right)$(Figure $\mathbf{4 E}$ and $\mathbf{F}$ ) in the superficial

164 layers of ipsilateral SDH in Mtor-cKO ${ }^{M G}$ mice. However, we were unable to observe

165 significant differences in mechanical allodynia (Figure 4G) or heat hyperalgesia

166 (Figure 4H) between the Mtor-cKO ${ }^{M G}$ and control mice after SNI (from day 1 to day

167 7), suggesting that neuropathic pain is spared in the absence of microglial mTOR

168 signaling.

169 Mtor ablation in DRG neurons suppressed elevation of subsets of nerve injury-

170 induced genes

171 To determine the downstream molecular targets of mTOR in DRG neurons involved in

172 neuropathic pain, we performed RNA sequencing of DRGs from Mtor ${ }^{f l f l}$ and Mtor- 
$c K O^{A d v}$ mice before and 7 days after SNI surgery. In total, the expression levels of 189 genes (155 upregulated and 34 downregulated), were significantly changed (by at least two folds, $p<0.05$ ) in the injured DRGs 7 days after SNI in Mtor ${ }^{f l / f l}$ mice (Figure 5AC). A large number of the upregulated genes, including those associated with injury (Activating transcription factor 3, Atf3 and Small proline-rich protein 1A, Sprr1a), Gprotein coupled receptors (Gpcrs, including Gpr151 and Gpr119), neuropeptides (Npy, Gal, and Nts), cytokines (Colony stimulating factor 1, Csf1 and Interleukin 1b, Il1b) have been previously reported in response to nerve injury (Figure 5B) (Wu et al., 2016; Reinhold et al., 2015; Guan et al., 2016; Peng et al., 2016), verifying the reliability of the RNA-seq data. Gene ontology analysis demonstrated that injury-affected genes were primarily enriched in four molecular functions (Figure 5C), including receptor ligand activity, hormone activity and neuropeptide receptor binding and activity.

Importantly, approximately 1/5 (32 in 155 genes) of injury-induced genes were suppressed after mTOR ablation (Figure 5E). In particular, the expression of two neuropeptide genes Npy and Nts, induced by approximately 73.5 and 11.7 folds after injury, was strikingly reduced to 3.75 and 0.57 folds after ablation of Mtor in DRG neurons. By contrast, expression of another two injury-induced neuropeptide genes, such as Corticotropin releasing hormone (Crh) and Gal remained largely unaffected, suggesting that mTOR specifically regulates the expression of subsets of injuryresponsive genes (Figure 5E-G). The reduced expression of Npy, Nts, and other genes (as indicated) in Mtor-cKO ${ }^{A d v}$ mice was further verified by qRT-PCR analysis (Figure 5-figure supplement 1). Notably, while mTOR was transiently activated during the 
195 first week after nerve injury, it may have long-term impacts on downstream molecules.

196 Collectively, these data demonstrate that mTOR regulates the transcription of a number

197 of injury-induced genes.

\section{Injury-activated mTOR is required for NPY induction in DRG neurons}

NPY is widely distributed in the central and peripheral nervous system (Allen et al.,

200 1983). It is absent in DRG neurons under homeostatic conditions but dramatically

201 upregulated after peripheral nerve injury (Wakisaka et al., 1991; Xiao et al., 2002).

202 However, nothing is known about the mechanisms regulating NPY induction after nerve injury. We also observed prominent induction of Npy in DRG neurons after nerve

204 injury, which lasted for at least 4 weeks with gradually reduced levels after day 14

205 (Figure 6A and B). Immunofluorescence analysis revealed that $94.2 \%$ of $\mathrm{NPY}^{+}$

206 neurons were co-labelled with ATF3, a marker for neuronal injury marker (Figure 6C

207 and D). Moreover, 89.6\% of $\mathrm{NPY}^{+}$neurons expressed p-S6 (Figure 6E and F), and

208 co-localized with NF160/200 in the injured DRGs (Figure 6-figure supplement 1),

209 suggesting that NPY is selectively induced in injured large-sized mechanoreceptors

210 with mTOR activation. It is noteworthy that Mtor ablation nearly eliminated NPY

211 induction (Figure 6E and G), indicating that mTOR inactivation suppressed NPY

212 transcription.

213 Previous studies indicated that the promoter regions of Npy and Nts genes harbor signal

214 transducer and activator of transcription 3 (STAT3)-binding site-like elements and that

215 dominant negative expression of STAT3 attenuated leptin-induced Npy and Nts 
Nerve injury-induced NPY enhances nociceptor excitability

NPY has been shown to increase the excitability of DRG neurons (Abdulla and Smith, 1999a). To examine whether mTOR-promoted NPY induction enhances the excitability of nociceptors, we carried out electrophysiological recording of small-sized nociceptors at 7 days SNI. As expected, nociceptors from $M$ tor $^{f l / f l}$ mice displayed increased number of action potentials and lower rheobase 7 days after SNI (Figure 7). By contrast, the

232 number of spikes was significantly reduced in mTOR-deficient neurons after injury. However, incubation of NPY with mTOR-deficient neurons significantly restored the number of action potentials and reduced rheobase, suggesting that NPY loss contributes

235 to the reduced nociceptor excitability in the absence of mTOR.

236 Studies have demonstrated different responses of DRG neurons to different NPY 
receptor agonists (Wiley et al., 1993; Abdulla and Smith, 1999b; Abdulla and Smith, 1999a). For example, Y2R agonists increased neuronal excitability of small DRG neurons, whereas Y1R agonists barely showed any effects (Abdulla and Smith, 1999a). We verified the distinct expression pattern of NPY and Y2R on large-sized mechanoreceptors and small-sized nociceptors by immunofluorescence analysis

(Figure 7-figure supplement 1) (Brumovsky et al., 2005). To determine which receptor mediates NPY-elicited excitatory effects, Y1R or Y2R antagonist was incubated with DRG neurons for 30 min before NPY addition. We found that blocking Y2R but not Y1R activity substantially reduced the number of action potentials after NPY addition, suggesting that Y2R mediates NPY-induced elevated excitation (Figure 7A-C).

\section{Peripheral NPY replenishment reversed analgesic effects of Mtor ablation through}

\section{Y2R}

NPY has been shown to elicit biphasic effects in pain processing by binding to different receptors in DRG or spinal neurons (Brumovsky et al., 2007). Given that mTOR ablation simultaneously delayed pain onset and suppressed NPY induction, we tested whether mTOR inactivation alleviated pain via NPY loss. We first administered a small dose of NPY (0.2 nmol), as previously suggested (Tracey et al., 1995; Brumovsky et al., 2005), into the hind paw of normal mice and observed prominent mechanical allodynia and heat hyperalgesia approximately 30 minutes after injection, supporting the pro-nociceptive effects of peripheral NPY (Figure 8A-C). By injecting NPY into 
258 the ipsilateral hind paw of Mtor-cKO ${ }^{A d v}$ mice, we observed robust mechanical allodynia,

259 and to a lesser extent, heat hyperalgesia in $M t o r-c K O^{A d v}$ mice (Figure 8E and F).

260 Moreover, blocking Y2R, rather than Y1R, before NPY administration substantially

261 reduced NPY-induced mechanical allodynia (Figure 8E), further supporting the role of

262 Y2R in mediating NPY-elicited pro-nociceptive effects. Collectively, our data

263 demonstrate that mTOR-induced NPY production in DRG neurons is essential for the

264 development of neuropathic pain via Y2R-mediated signaling. 


\section{DISCUSSION}

Neuropathic pain is a maladaptive response of the nociceptive pathway to the nerve injury. Both peripheral and central sensitization have been shown to contribute to the persistent pain (Colloca et al., 2017). Peripheral nociceptor sensitization is a key trigger in neuropathic pain, as inhibiting nociceptor activity by anesthetics effectively blocks pain (Colloca et al., 2017). In this study, we uncover a previously unrecognized mechanism, by which injury-induced mTOR activation and downstream transcriptional factor STAT3 drives NPY synthesis to enhance nociceptor excitability and promote pain development through Y2R. Considering the distinct distribution patterns of NPY and Y2R in large-sized mechanoreceptors and small-sized nociceptors, mTOR-driven pain may involve an intra-ganglia communication between NPY-expressing mechanoreceptors and Y2R-expressing nociceptors.

Basal levels of mTOR activity are present in a small subset of large-sized myelinated sensory neurons in naïve mice (Lisi et al., 2015; Geranton et al., 2009). In the present study, we observed increased mTOR activation predominantly occurs in large sensory neurons and spinal microglia after nerve injury. While pharmacologically blocking mTOR activity has raised controversies regarding its role in pain (Obara et al., 2011; Geranton et al., 2009; Melemedjian et al., 2013; Khoutorsky et al., 2015), we found that selective ablation of mTOR in primary sensory neurons, which disrupted both mTORC1 and mTORC2, robustly prevented the early onset of nerve injury-triggered allodynia and heat hyperalgesia for 2 weeks. Selective ablation of Raptor or Rictor in 
DRG neurons are needed to distinguish roles of mTORC1 and mTORC2 signaling in neuropathic pain (Laplante and Sabatini, 2012). In contrast to the ERK activation

288 following mTORC1 inhibition as previously described (Melemedjian et al., 2013), we did not observe ERK activation in DRG neurons (data not shown) after genetic inactivation of mTOR in SNI models. The delayed onset of mechanical allodynia after mTOR ablation are in line with the temporal activation of mTOR and expression of 292 downstream effectors after nerve injury, emphasizing a central role of mTOR in 293 promoting neuropathic pain development. Additional maladaptive changes other than mTOR signaling may contribute to the late-phase pain.

Nerve injury-induced de novo synthesis of a large number of molecules are implicated in the hypersensitive nociception (Wang et al., 2021; Zhao et al., 2017). For example,

297 injury-induced CSF1 in DRG neurons, a cytokine required for microglial and 298 macrophage expansion, has recently been shown to contribute to mechanical hypersensitivity (Yu et al., 2020; Guan et al., 2016; Peng et al., 2016). Also, removal of the eukaryotic initiation factor 4E-binding protein 1 (4E-BP1), a negative regulator 301 of protein translation downstream of mTOR, induced pain hypersensitivity through 302 enhanced translation of neuroligin 1 even in the absence of nerve injury, further 303 stressing the importance of mTOR-mediated protein synthesis in pain hypersensitivity 304 (Khoutorsky and Price, 2018; Khoutorsky et al., 2015; Yousuf et al., 2020). Our 305 findings that mTOR is required for nerve injury-induced Npy and Nts transcription demonstrate novel links between mTOR activation to neuropeptide production. 

control, mTOR is unlikely to directly promote Npy or Nts transcription. In search for potential mTOR-regulated transcriptional factors upstream of Npy or Nts genes, we observed suppressed phosphorylation of STAT3, but not C-Jun or CREB (data not shown), in DRG neurons after mTOR deletion. Since STATs-like binding elements are present in the promoter region of Npy gene (Muraoka et al., 2003), it is likely that activated mTOR induces Npy transcription by phosphorylating STAT3, thereby promoting STAT3 nuclear entry and downstream gene transcription. While previous studies primarily suggested that mTOR contributes to pain sensitivity through translational control (Khoutorsky and Price, 2018; Melemedjian and Khoutorsky, 2015), our study demonstrate a non-translational mechanism of mTOR involving STAT3NPY production in pain regulation.

Nerve injury often induces nociceptor hyper-excitability to provoke pain hypersensitivity. However, this hyper-excitability was lost after ablation of mTOR in DRG neurons, along with elimination of NPY. NPY has been shown to elicit both antinociceptive and pro-nociceptive effects, depending on the subtypes of its receptors in the central and peripheral nervous system (Brumovsky et al., 2007; Diaz-delCastillo et al., 2018). We found that NPY was selectively induced in injured large-sized sensory neurons, suggesting the peripheral effects of NPY. In contrast to previous studies showing that NPY triggers analgesia by inhibiting superficial dorsal horn interneurons through Y1R (Taiwo and Taylor, 2002; Miyakawa et al., 2005; Nelson and Taylor, 
Replenishing NPY enhanced nociceptor excitability, while peripherally blocking Y2R, but not Y1R, prevented these effects, suggesting that mTOR drives NPY production to enhance nociceptor excitability through Y2R. Consistent with our observations, a previous study indicated that $\mathrm{Y} 1 \mathrm{R}$ agonist had no effect on small DRG neurons, whereas Y2R agonist enhanced neuronal excitability (Abdulla and Smith, 1999a). A reasonable explanation is that $\mathrm{Y} 2 \mathrm{R}$ attenuated calcium-sensitive potassiumconductance, thereby inducing nociceptor depolarization and excitability (Abdulla and Smith, 1999a; Abdulla and Smith, 1999b).

It is noteworthy that NPY elevation is exclusively observed in large-diameter mechanoreceptors, whereas $\mathrm{Y} 2 \mathrm{R}$ is predominantly distributed in small-diameter nociceptors (Brumovsky et al., 2005). The distinct but adjacent distribution of NPY suggests a paracrine ‘somatic cross excitation' model within DRGs, by which NPY

341 released from the large-diameter injured neurons acts on neighboring small-diameter Y2R-expressing neurons (Brumovsky et al., 2007). Through intra-ganglionic transmission (Brumovsky et al., 2007), NPY signals derived from large injured mechanoreceptors are able to sensitize Y2R-expressing nociceptors, thereby

345 contributing to mechanical allodynia. In line with this concept, blocking Y2R effectively alleviated mechanical allodynia. Our findings thus provide an important mechanism for mechanical allodynia engaging mTOR-driven NPY-Y2R communication between mechanoreceptors and nociceptors in neuropathic pain. Other than NPY, mTOR-driven expression of NTS and GPCRs likely coordinately contribute 
351 Microglia activation in SDH have been shown to contribute to neuropathic pain (Inoue

352 and Tsuda, 2018). Moreover, mTOR-mediated metabolic reprogramming are required

353 for induction of inflammatory factors and cytokines in microglia (Hu et al., 2019),

354 which indicated that mTOR activation in microglia may be involved in neuropathic

355 pain. We found that Mtor deletion in microglia reduced microgliosis; however, it did

356 not have significant effects on neuropathic pain. This is likely due to the fact that mTOR

357 was activated in less than $50 \%$ of microglia in the SDH and that mTOR ablation only

358 partially reduced microgliosis, which might be insufficient to inhibit pain development.

359 Consistent with this notion, removal of microglia only ameliorated mechanical

360 allodynia during the first 3 days after nerve injury, whereas removal of both microglia

361 and peripheral monocytes/macrophage prevented neuropathic pain development (Peng

362 et al., 2016).

363 In summary, we demonstrate that nerve injury-induced aberrant mTOR activation in 364 sensory neurons promotes pain development. While mTOR has been shown to affect 365 the expression or function of hundreds of molecules, the present study is the first that 366 links mTOR to NPY signaling in sensitizing nociceptive pathway to drive neuropathic 367 pain. As mTOR inhibitors are in clinical use and Y2R receptor antagonists are readily 368 available, our findings also provide new perspectives for clinically treating neuropathic pain by peripherally modulating mTOR and NPY-Y2R signaling. 


\begin{tabular}{|c|c|c|c|}
\hline $\begin{array}{l}\text { Reagent type } \\
\text { (species) or resource }\end{array}$ & Designation & Source or reference & Identifiers \\
\hline Antibody & $\begin{array}{l}\text { Rabbit anti-p-S6 ribosomal } \\
\text { protein-Ser } 235 / 236\end{array}$ & Cell Signaling Technology & $\begin{array}{l}\text { Cat\# 4858; RRID: } \\
\text { AB_916156 }\end{array}$ \\
\hline Antibody & $\begin{array}{l}\text { Rabbit anti-S6 ribosomal } \\
\text { protein }\end{array}$ & Cell Signaling Technology & $\begin{array}{l}\text { Cat\# 2217; RRID: } \\
\text { AB_331355 }\end{array}$ \\
\hline Antibody & Rabbit anti-NPY & Cell Signaling Technology & $\begin{array}{l}\text { Cat\# 11976; RRID: } \\
\text { AB_2716286 }\end{array}$ \\
\hline Antibody & Rabbit anti-p-STAT3-Ser ${ }^{727}$ & Cell Signaling Technology & $\begin{array}{l}\text { Cat\# 9134; RRID: } \\
\text { AB_331589 }\end{array}$ \\
\hline Antibody & Rabbit anti-NPY Y2 receptor & Neuromics & $\begin{array}{l}\text { Cat\# RA14112; RRID: } \\
\text { AB_2315615 }\end{array}$ \\
\hline Antibody & $\begin{array}{l}\text { Rat anti-5-Bromo-2'- } \\
\text { deoxyuridine }\end{array}$ & Abcam & $\begin{array}{l}\text { Cat\# ab6326; RRID: } \\
\text { AB_305426 }\end{array}$ \\
\hline Antibody & Goat anti-GFP & Abcam & $\begin{array}{l}\text { Cat\# ab5450; RRID: } \\
\text { AB_304897 }\end{array}$ \\
\hline Antibody & Mouse anti- $\beta$-Actin & Sigma-Aldrich & $\begin{array}{l}\text { Cat\# A1978; RRID: } \\
\text { AB_476692 }\end{array}$ \\
\hline Antibody & $\begin{array}{l}\text { Mouse anti-Neurofilament } \\
160 / 200\end{array}$ & Sigma-Aldrich & $\begin{array}{l}\text { Cat\# n2912; RRID: } \\
\text { AB_477262 }\end{array}$ \\
\hline Antibody & Mouse anti-GFAP & Synaptic Systems & $\begin{array}{l}\text { Cat\# 173011; RRID: } \\
\text { AB_2232308 }\end{array}$ \\
\hline Antibody & Mouse anti-NeuN & Millipore & $\begin{array}{l}\text { Cat\# MAB377; RRID: } \\
\text { AB_2298772 }\end{array}$ \\
\hline Antibody & Rabbit anti-Iba1 & Wako & $\begin{array}{l}\text { Cat\# 019-19741; } \\
\text { RRID: AB_839504 }\end{array}$ \\
\hline Antibody & Mouse anti-p-S6-Ser ${ }^{235 / 236}$ & Santa Curz Biotechnology & Cat\# sc-293144 \\
\hline Antibody & Mouse anti-CGRP & Santa Curz Biotechnology & $\begin{array}{l}\text { Cat\# sc-57053; RRID: } \\
\text { AB_2259462 }\end{array}$ \\
\hline Antibody & Mouse anti-ATF3 & Santa Curz Biotechnology & $\begin{array}{l}\text { Cat\# sc-81189; RRID: } \\
\text { AB_2058591 }\end{array}$ \\
\hline Antibody & $\begin{array}{l}\text { Alexa Fluor 488-donkey anti- } \\
\text { goat }\end{array}$ & Invitrogen, Thermo Fisher & $\begin{array}{l}\text { Cat\# A11055; RRID: } \\
\text { AB_2534102 }\end{array}$ \\
\hline Antibody & $\begin{array}{l}\text { Alexa Fluor 488-donkey anti- } \\
\text { rabbit }\end{array}$ & Invitrogen, Thermo Fisher & $\begin{array}{l}\text { Cat\# A21206; RRID: } \\
\text { AB_2535792 }\end{array}$ \\
\hline Antibody & $\begin{array}{l}\text { Alexa Fluor 488-donkey anti- } \\
\text { mouse }\end{array}$ & Invitrogen, Thermo Fisher & $\begin{array}{l}\text { Cat\# A21202; RRID: } \\
\text { AB_141607 }\end{array}$ \\
\hline Antibody & $\begin{array}{l}\text { Alexa Fluor 555-donkey anti- } \\
\text { mouse }\end{array}$ & Invitrogen, Thermo Fisher & $\begin{array}{l}\text { Cat\# A31570; RRID: } \\
\text { AB_2536180 }\end{array}$ \\
\hline Antibody & $\begin{array}{l}\text { Alexa Fluor 555-donkey anti- } \\
\text { rabbit }\end{array}$ & Invitrogen, Thermo Fisher & $\begin{array}{l}\text { Cat\# A31572; RRID: } \\
\text { AB_162543 }\end{array}$ \\
\hline Antibody & Cy3-donkey anti-rat & Jackson ImmunoResearch & $\begin{array}{l}\text { Cat\# 712-165-153; } \\
\text { RRID: AB_2340667 }\end{array}$ \\
\hline
\end{tabular}


bioRxiv preprint doi: https:/doi.org/10.1101/2021.10.29.466458; this version posted November 1, 2021. The copyright holder has placed this preprint (which was not certified by peer review) in the Public Domain. It is no longer restricted by copyright. Anyone can legally share, reuse, remix, or adapt this material for any purpose without crediting the original authors.

\begin{tabular}{|c|c|c|c|}
\hline Antibody & $\begin{array}{l}\text { Horseradish peroxidase } \\
\text { (HRP)-conjugated goat anti- } \\
\text { rabbit }\end{array}$ & Jackson ImmunoResearch & $\begin{array}{l}\text { Cat\# 111-035-003; } \\
\text { RRID: AB_2313567 }\end{array}$ \\
\hline Antibody & $\begin{array}{l}\text { Horseradish peroxidase } \\
\text { (HRP)-conjugated goat anti- } \\
\text { mouse }\end{array}$ & Jackson ImmunoResearch & $\begin{array}{l}\text { Cat\# 115-035-146; } \\
\text { RRID: AB_2307392 }\end{array}$ \\
\hline $\begin{array}{l}\text { peptide, recombinant } \\
\text { protein }\end{array}$ & NPY & Tocris & Cat\# 1153 \\
\hline $\begin{array}{l}\text { peptide, recombinant } \\
\text { protein }\end{array}$ & scrambled NPY & Tocris & Cat\# 3903 \\
\hline $\begin{array}{l}\text { chemical compound, } \\
\text { drug }\end{array}$ & BIBO3304 trifluoroacetate & Tocris & $\begin{array}{l}\text { Cat\# 2412; CAS: } \\
\text { 191868-14-1 }\end{array}$ \\
\hline $\begin{array}{l}\text { chemical compound, } \\
\text { drug }\end{array}$ & BIIE0246 & Tocris & $\begin{array}{l}\text { Cat\# 1700; CAS: } \\
\text { 246146-55-4 }\end{array}$ \\
\hline $\begin{array}{l}\text { chemical compound, } \\
\text { drug }\end{array}$ & BIIE0246 hydrochloride & Tocris & Cat\# 7377 \\
\hline $\begin{array}{l}\text { chemical compound, } \\
\text { drug }\end{array}$ & $\begin{array}{l}\text { Isolectin GS-IB4, Alexa } \\
\text { FluorTM } 568 \text { Conjugate }\end{array}$ & Invitrogen, Thermo Fisher & Cat\# I21412 \\
\hline $\begin{array}{l}\text { chemical compound, } \\
\text { drug }\end{array}$ & Rapamycin & Selleck Chemicals & $\begin{array}{l}\text { Cat\# S1039; CAS: } \\
\text { 53123-88-9 }\end{array}$ \\
\hline $\begin{array}{l}\text { chemical compound, } \\
\text { drug }\end{array}$ & 5-Bromo-2'-deoxyuridine & Sigma-Aldrich & $\begin{array}{l}\text { Cat\#: 19-160; CAS: } \\
59-14-3\end{array}$ \\
\hline $\begin{array}{l}\text { Commercial assay or } \\
\text { kit }\end{array}$ & $\begin{array}{l}\text { Multiple-color } \\
\text { immunochemistry kit }\end{array}$ & Absin & Cat\# abs50012 \\
\hline $\begin{array}{l}\text { Commercial assay or } \\
\text { kit }\end{array}$ & RNeasy micro kit & Qiagen & Cat\# 74004 \\
\hline $\begin{array}{l}\text { Commercial assay or } \\
\text { kit }\end{array}$ & PrimeScript RT Reagent Kit & TaKaRa & Cat\# RR037A \\
\hline $\begin{array}{l}\text { Commercial assay or } \\
\text { kit }\end{array}$ & SYBR Premix Ex Taq ${ }^{\mathrm{TM}}$ & TaKaRa & Cat\# DRR041A \\
\hline $\begin{array}{l}\text { Strain, strain } \\
\text { background (Mus } \\
\text { musculus) }\end{array}$ & C57BL/6J & $\begin{array}{l}\text { Shanghai Slac Laboratory } \\
\text { Animal Corporation }\end{array}$ & Mouse: C57BL/6J \\
\hline $\begin{array}{l}\text { Strain, strain } \\
\text { background (Mus } \\
\text { musculus) }\end{array}$ & $\begin{array}{l}\text { Cx3cr1 }{ }^{\text {EGFP/+}: ~ B 6.129 P-~} \\
\text { Cx3cr1 }{ }^{\text {tm1Litt/J }}\end{array}$ & The Jackson Laboratory & Stock\#: 005582 \\
\hline $\begin{array}{l}\text { Strain, strain } \\
\text { background (Mus } \\
\text { musculus) } \\
\end{array}$ & $\begin{array}{l}\text { Mtor }{ }^{\mathrm{fl} / \mathrm{fl}:} \text { B6.129S4- } \\
\text { Mtor }^{\mathrm{tm} 1.2 \mathrm{Koz} / \mathrm{J}}\end{array}$ & The Jackson Laboratory & Stock\#: 011009 \\
\hline $\begin{array}{l}\text { Strain, strain } \\
\text { background (Mus } \\
\text { musculus) } \\
\end{array}$ & $\begin{array}{l}\text { Advillin' }^{\text {cree }} \text { B6.129P2- } \\
\text { Avil }^{\text {tm2(cre)Fawa/J }}\end{array}$ & The Jackson Laboratory & Stock\#: 032536 \\
\hline
\end{tabular}


bioRxiv preprint doi: https://doi.org/10.1101/2021.10.29.466458; this version posted November 1, 2021. The copyright holder has placed this preprint (which was not certified by peer review) in the Public Domain. It is no longer restricted by copyright. Anyone can legally share, reuse, remix, or adapt this material for any purpose without crediting the original authors.

\begin{tabular}{|c|c|c|c|}
\hline $\begin{array}{l}\text { Strain, strain } \\
\text { background (Mus } \\
\text { musculus) }\end{array}$ & $\begin{array}{l}\text { Cx3cr1 }{ }^{\text {creER/+ }}: \text { B6.129P2(Cg)- } \\
\text { Cx3cr1 } 1^{\text {tm2.1(cre/ERT2)Litt/WganJ }}\end{array}$ & The Jackson Laboratory & Stock\#: 011009 \\
\hline $\begin{array}{l}\text { Strain, strain } \\
\text { background (Mus } \\
\text { musculus) }\end{array}$ & $\mathrm{Cx}^{2} \mathrm{cr}^{\mathrm{creER} /+}:: \mathrm{Mtor}^{\mathrm{fl} / \mathrm{fl}}$ & This paper & N/A \\
\hline $\begin{array}{l}\text { Strain, strain } \\
\text { background (Mus } \\
\text { musculus) }\end{array}$ & Advillin ${ }^{\text {cre.:: Mtor }}{ }^{\mathrm{fl} / \mathrm{fl}}$ & This paper & N/A \\
\hline $\begin{array}{l}\text { Sequence-based } \\
\text { reagent }\end{array}$ & $\begin{array}{l}\text { Primers used for Mtor }{ }^{\text {fl/fl: }} \\
\text { Mtor-P1 (5'- } \\
\text { GCTCTTGAGGCAAATGC } \\
\text { CACTATCACC-3'), Mtor-P2 } \\
\text { (5'- TCATTACC } \\
\text { TTCTCATCAGCCAGCAGT } \\
\text { T-3'), Mtor-P3 (5'- } \\
\text { TTCATTCCCTTGAAAGCC } \\
\text { AGTCTCACC-3') }\end{array}$ & This paper & $\mathrm{N} / \mathrm{A}$ \\
\hline $\begin{array}{l}\text { Sequence-based } \\
\text { reagent }\end{array}$ & $\begin{array}{l}\text { Primers for RT-qPCR, see } \\
\text { Table } 1\end{array}$ & This paper & N/A \\
\hline Software, algorithm & ImageJ & $\mathrm{NIH}$ & $\begin{array}{l}\text { https://imagej.nih.gov/i } \\
\text { j }\end{array}$ \\
\hline Software, algorithm & ANY-maze & Stoelting & $\begin{array}{l}\text { https://www.anymaze. } \\
\text { co.uk/index.htm }\end{array}$ \\
\hline Software, algorithm & pClamp & Molecular Devices & $\begin{array}{l}\text { https://www.molecular } \\
\text { devices.com/products/ } \\
\text { axonpatch-clamp- } \\
\text { system/acquisition- } \\
\text { and- } \\
\text { analysissoftware/pcla } \\
\text { mp-software-suite }\end{array}$ \\
\hline Software, algorithm & Prism & GraphPad & $\begin{array}{l}\text { https://www.graphpad. } \\
\text { com/scientificsoftware } \\
\text { /prism }\end{array}$ \\
\hline Software, algorithm & $\mathrm{R}$ & R Foundation & $\begin{array}{l}\text { https://www.r- } \\
\text { project.org/ }\end{array}$ \\
\hline
\end{tabular}


Adult male mice (8-12 weeks) were used for biochemical and behavioral tests, and young mice (4-6 weeks) for whole-cell patch clamp recording. C57BL/6J mice were

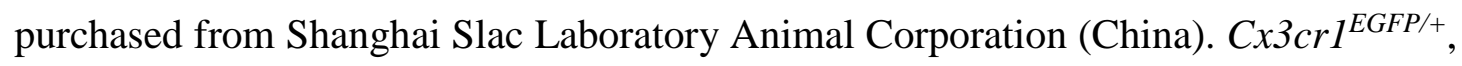
CX3cr $1^{\text {creER/+}}$, Mtor floxed $\left(M t o r^{f l / f l}\right)$ and Advillin ${ }^{\text {cre }}\left(A d v^{c r e}\right)$ mice with C57BL/6J background were purchased from the Jackson Laboratory (ME, USA). All animals were housed under a 12-h light/dark cycle with food and water available. To selective knockout the Mtor gene in microglia, mice bearing the floxed allele of the Mtor gene $\left(M_{t o r}{ }^{f l / f l}\right)$ were crossed with $C x 3 c r 1^{c r e E R /+}$ mice. $C x 3 c r 1^{c r e E R /+}:: M t o r^{f l / f l}$ mice received two doses of $10 \mathrm{mg}$ tamoxifen citrate (TAM, Meilunbio, China) or vehicle in 48-hour intervals. TAM induced the expression of Cre recombinase in both resident microglia and peripheral monocytes. Since monocytes have a rapid turnover rate, Cre expression is eliminated in peripheral monocytes but maintained in resident microglia 4-6 weeks after TAM induction (Parkhurst et al., 2013), thus allowing selective deletion of Mtor in microglia (Mtor-cKO ${ }^{M G}$ ) but not in monocytes. Control mice were $C x 3 c r 1^{c r e E R /+}:: M t o r r^{f l / f l}$ littermates without TAM induction and $C x 3 c r 1^{c r e E R /+}$ mice with TAM induction. For selective ablation of Mtor in DRG sensory neurons, Mtor ${ }^{f l f l}$ mice were crossed with $A d v^{c r e}$ mice to obtain the $A d v^{c r e}:: M t o r^{f l / f l}$ (Mtor-cKO ${ }^{A d v}$ ) mice. Mtor$c K O^{A d v}$ mice enabled Mtor deletion in DRG neurons but leave spinal cord unaffected. Control mice were Mtor ${ }^{f l / f l}$ littermates without Cre promotor.

\section{Cre-mediated recombination of the $M$ tor $^{f l o x}$ allele}

Primers used for analyses of Mtor floxed alleles were as the following: Mtor-P1 (5'GCTCTTGAGGCAAATGCCACTATCACC-3’), Mtor-P2 (5’- $\quad$ TCATTACC 
AGTCTCACC-3’). Primer pair P1/P2 was used for genotyping floxed mTOR alleles

that generated a 480 bp DNA fragment in PCR (Risson et al., 2009). Upon Cre-

\section{Neuropathic pain model}

Spared nerve injury (SNI) models were used to induce neuropathic pain as previously

described (Decosterd and Woolf, 2000). Mice were anesthetized with sodium pentobarbital (100 mg/kg) intraperitoneally. The left hindlimb was shaved, and the skin

409 ligated with 5-0 silk sutures, with care to avoid injury to the sural nerve. The ligated

410 branches were then transected distal to the ligature and a 2-3 $\mathrm{mm}$ distal nerve stump

411 was removed. To minimize the number of animals used in the experiments, the right

413 ligation and transection. To analyze NPY transport from the DRG to the spinal cord,

414 we ligated the ipsilateral L4 central axonal branches immediately after SNI. After the

415 surgery, the incision was closed using 5-0 silk sutures. The injured side was then 416 regarded as the ipsilateral side, and the uninjured as the contralateral one. 

isolated at certain time points after SNI surgery, snap-frozen in liquid nitrogen and stored at $-80^{\circ} \mathrm{C}$. Tissues were homogenized in RIPA lysis buffer (Beyotime, China) with protease inhibitor (Cat\# S8830, Sigma-Aldrich, MO, USA) and phosphatase inhibitor (Cat\# A32961, Thermo Fisher, MA, USA) using ultrasonic cell disruptor. The homogenates were centrifuged at $4{ }^{\circ} \mathrm{C}$ for 30 minutes at $10,000 \mathrm{~g}$ and the supernatants

424 were collected. Proteins were separated by 10\% SDS-polyacrylamide gels and 425 transferred to polyvinylidene difluoride membranes (Millipore, Germany), followed by blocking, primary antibodies and horseradish peroxidase (HRP)-conjugated secondary

427 antibodies (1:10000, Jackson ImmunoResearch, PA, USA) incubation. The proteins 428 were detected using enhanced chemiluminescence regents (ECL, Amersham Pharmacia Biotech, NJ, USA) according to the manual.

\section{Immunofluorescence analysis}

After deeply anaesthetized with sodium pentobarbital, mice were perfused with saline and subsequently 4\% paraformaldehyde (PFA, Sigma-Aldrich). The spinal cord and L4-L5 DRGs were dissected, post-fixed in 4\% PFA, and transferred to 30\% sucrose in 0.1 M phosphate buffer ( $\mathrm{pH}=7.2$ ) for 2 days. Samples were embedded in optimal cutting temperature (OCT) and transverse sections were cut using freezing microtome (Lecia neurons in DRG, slices were blocked with 10\% (wt/vol) normal bovine serum albumin (BSA) for 1 hour at room temperature, and incubated with $1 \mu \mathrm{g} / \mathrm{mL}$ IB4 diluted in 
with Tris buffered saline (TBS) and then incubated with anti-p-S6 (1:1000) antibody. For staining with other antibodies, sections were antigen-retrieved in citrate buffer (10 mM sodium citrate, $0.05 \%$ Tween-20, $\mathrm{pH}$ 6.0) or Tris-EDTA (10 mM Tris, $1 \mathrm{mM}$ EDTA, 0.05\% Tween-20, $\mathrm{pH}$ 9.0) as appropriate at $95{ }^{\circ} \mathrm{C}$ for 20 minutes and permeabilized with $0.5 \%$ Triton $\mathrm{X}-100$ for 10 minutes at room temperature. After blocked with $10 \%$ (wt/vol) BSA, sections were incubated overnight at $4{ }^{\circ} \mathrm{C}$ with following primary antibodies: rabbit anti-p-S6 (1:1000), mouse anti-p-S6 (1:2000 1:4000), mouse anti-NeuN (1:1000), mouse anti-NF160/200 (1:2000), mouse antiCGRP (1:1000), rat anti-BrdU (1:800), and goat anti-GFP (1:1000), rabbit anti-Iba1 (1:800), rabbit anti-NPY (1:1000), mouse anti-GFAP (1:800), mouse anti-ATF3 (1:200), rabbit anti-p-STAT3 (Ser727) (1:500). Sections were then washed in TBS with 0.5\% tween (TBS-T) and incubated with appropriate secondary antibodies (1:1000) for 1.5 hours at room temperature. For NPY and Y2R staining, since both anti-NPY and Y2R antibodies were raised in rabbits, the multiple-color immunochemistry kit (Cat\# abs50012, Absin, China) was used following the manufacturer's instructions. The specificity of the staining using this kit was first validated by double staining of rabbit anti-NPY and Iba1 antibodies that showed no overlaps. Following the anti-NPY incubation, rabbit horseradish peroxidase (HRP)-conjugated secondary antibody (1:1000) was applied and incubated for 1.5 hours. Sections were than washed in TBST and incubated with Tyramide Signal Amplification (TSA) reagent for 10 minutes. Antibody eluent (Cat\# abs994, Absin) was used to wash out anti-NPY and HRPconjugated antibody. After washing, sections were incubated with anti-Y2R antibody 
462

463

464

(1:500) and followed by incubation with appropriate secondary antibodies (1:1000) according to species of the first antibody. DAPI (Beyotime) was used to label cell nuclei in tissue sections. The immunofluorescence images were captured by FV-1200 confocal microscope (Olympus, Japan). The density or percentage of positive cells in SDH and DRGs were counted and calculated using 3 sections from each animal. Mean intensity of interested regions were evaluated using ImageJ software.

\section{Drug administration}

BrdU was used to label proliferating cells in the spinal cord after the SNI surgery. The BrdU labeling procedure was carried out as described before (Gu et al., 2016), with two intraperitoneal injections (100 mg/kg) daily one day before the surgery until 7 days post-surgery. For intraperitoneal treatment of rapamycin, mice were administrated with rapamycin (5 mg/kg) or vehicle daily one day before SNI until 7 days post-surgery. For local intraplantar (i.pl.) injection, drugs (0.2 nmol NPY, $0.2 \mathrm{nmol}$ scrambled NPY, 5 nmol BIBO3304 trifluoroacetate or $50 \mathrm{nmol}$ BIIE0246) in $20 \mu 1$ saline were injected using a syringe with a 30-gauge needle. Dosages of NPY and its antagonists were referred to the previous studies (Tracey et al., 1995; Sapunar et al., 2011). NPY receptor antagonists were injected 1 hour before NPY injection. To assess the effects after i.pl. injection, behavioral tests were finished in 30-40 minutes after NPY or scrambled peptide injection. The von Frey and Hargreaves tests were used for an interval of at least 4 hours.

\section{RNA sequencing}


Bilateral SNI were performed in $M t o r^{f l / f l}$ and Mtor-cKO ${ }^{A d v}$ mice to minimize the animals used in the experiment. In total, 4 lumber DRGs (bilateral L4 and L5 DRGs) were collected from each mouse before or 7 days after SNI. RNAs were isolated using RNeasy micro kit (Cat\# 74004, QIAGEN, Germany) according to the manufacturer's instructions. RNA sequencing (RNA-seq) libraries were constructed and sequenced by BGISEQ-500 (BGI, China). After quality control, the raw RNA-seq data were filtered to obtain the clean data used for alignment to the mouse genome (Mus musculus GRCm38.p5, NCBI). Based on these read counts, normalization and differential gene expression were performed using DESeq2 on $R$ (version 3.5.3). Genes with fragments per kilobase million $(\mathrm{FPKM})$ lower than $1(\mathrm{FPKM}<1)$ in all groups were excluded from the subsequent analyses. Statistical significance of differentially expressed genes (DEGs) was calculated based on the raw counts of individual genes, with an absolute fold change greater than 2 and adjusted p-value (q-value) less than 0.05.

Volcano plots and heatmaps were visualized by R (the ggplot2 and gplots packages, respectively). Gene Ontology (GO) enrichment in the molecular function category were visualized by R (bioconductor package "org.Hs.eg.db" and "cluster profiler" package).

\section{Quantitative RT-PCR}

Total RNA from DRG was extracted using RNeasy micro kit and reverse-transcribed using PrimeScript RT Reagent Kit (Cat\# RR037A, TaKaRa, Japan). Real-time PCR was performed using the SYBR Premix Ex Taq ${ }^{\mathrm{TM}}$ (Cat\# DRR041A, Takara) on a LightCycler 480 Instrument II Real-Time PCR Detection System (Roche). Primer 
504

505

506

507

508

509

sequences are provided in the Table 1. The relative expression was measured using the $2^{-\Delta \Delta \mathrm{Ct}}$ method. Briefly, the threshold cycle $(\mathrm{Ct})$ values of target genes were determined automatically by LightCycler $480 \mathrm{II}$ software. $\Delta \mathrm{Ct}=\mathrm{Ct}_{\text {(Target genes) }}-\mathrm{Ct}_{\beta \text {-actin. }} \Delta \Delta \mathrm{Ct}=$ $\Delta \mathrm{Ct}_{\text {(Target genes) }}-\Delta \mathrm{Ct}_{\text {(average } \Delta \mathrm{Ct} \text { of control). }}$ Relative fold changes were determined by $2^{-\Delta \Delta \mathrm{Ct}}$ and normalized to the expression levels of Actin (Livak and Schmittgen, 2001).

\section{Whole-cell patch clamp recording}

Mice were anesthetized with sodium pentobarbital before sterilized with 75\% alcohol. L4 and L5 DRGs were carefully collected on ice and digested with collagenase IV (0.2 mg/mL, Cat\# LS004188, Worthington, NJ, USA) and dispase-II (3 mg/mL, Cat\# D4693, Sigma-Aldrich) for $60 \mathrm{~min}$ at $37^{\circ} \mathrm{C}$. The cell suspension was centrifuged at $500 \mathrm{~g}$ for $10 \mathrm{~min}$ through a cushion of 15\% BSA (Cat\# A9205, Sigma-Aldrich) in order to eliminate most of the cellular debris. The cell pellet was resuspended in Neurobasal medium (Cat\# 21103049, Thermo Fisher Scientific) with B27 (Cat\# 17504-044, Invitrogen, Thermo Fisher Scientific) and NGF supplement (50 ng/mL, Cat\# 13257019, Gibco, Thermo Fisher Scientific) and seeded onto glass coverslips coated with poly-D-lysine (Cat\# P7280, Sigma-Aldrich) and cultured in $5 \% \mathrm{CO}_{2}$ incubator at $37^{\circ} \mathrm{C}$ for at least 2 h before recording. For drug treatment, cultured DRG neurons were incubated with $300 \mathrm{nM}$ NPY for 30 min before recording. To antagonize Y1R or Y2R, BIBO3304 trifluoroacetate (BIBO3304, $1 \mu \mathrm{M})$ or BIIE0246 hydrochloride $(1 \mu \mathrm{M})$ was replenished respectively into medium 30 min before NPY addition.

Whole-cell patch clamp recordings were carried out at room temperature using a 
525 Multiclamp 700B amplifier (Molecular Devices, CA, USA). The resistances of

526 borosilicate glass electrodes were measured ranging from 3 to $5 \mathrm{M} \Omega$. The intracellular

527 pipette solution contained (in mM) $135 \mathrm{~K}$-gluconate, $6 \mathrm{NaCl}, 10 \mathrm{HEPES}, 0.5$ EGTA,

$52810 \mathrm{Na}_{2}$-phosphocreatine, $4 \mathrm{Mg}$-ATP, $0.3 \mathrm{Na}_{2}-\mathrm{GTP}$, and was adjusted to $\mathrm{pH} 7.2$ using

$529 \mathrm{KOH}$. The extracellular solution was composed of (in $\mathrm{mM}$ ) $150 \mathrm{NaCl}, 5 \mathrm{KCl}, 2.5 \mathrm{CaCl}_{2}$,

$5301 \mathrm{MgCl}_{2}, 10$ HEPES, and 10 glucose, and was adjusted to $\mathrm{pH} 7.4$ by $\mathrm{NaOH}$. Action

531 potential firing and resting membrane potential (RMP) were recorded from small-

532 diameter neurons $(<20 \mu \mathrm{m})$. Data were collected from neurons with stable RMP

533 negative than $-40 \mathrm{mV}$. Action potentials were evoked by current injection steps. Data

534 were digitized with Digidata 1440A (Molecular Devices), and analyzed by pClamp

535 software (Version 10.6, Molecular Devices).

536 Behavioral tests

537 The following behavioral tests were conducted in a blinded manner and during daytime

538 (light cycle). For all experiments, experimenters were blinded to genotypes or 539 experimental manipulation. All the apparatuses and cages were sequentially wiped with

$540 \quad 70 \%$ ethanol and $\mathrm{ddH}_{2} \mathrm{O}$ then air-dried between stages.

541 von Frey tests

542 von Frey tests were used to evaluate 50\% paw withdrawal threshold (50\% PWT) during

543 the light cycle. In brief, individual mouse was habituated in an opaque plexiglas

544 chamber on a wire mesh platform for 30 minutes prior to test. Testing was performed

545 using a set of von Frey filaments (0.008-2 g, North Coast Medical, CA, USA). Each 
546 filament was applied to the lateral part of plantar surface of the mouse hind paw

547 vertically for up to 3 seconds from the bottom. Positive response was determined as a

548 sharp withdrawal, shaking or licking of the limb. The 50\% PWT was determined by the

549 up-down method (Dixon, 1965). Test was carried out at 1 day before SNI (baseline)

550 and at $1,3,5$, and 7 days post-surgery.

553 Science, CA, USA). The basal paw withdrawal latency was adjusted to 9-12 s, with a

554 cutoff of $20 \mathrm{~s}$ to avoid tissue damage.

Mice were placed on the hot plate (IITC Life Science) at 50,52 or $56^{\circ} \mathrm{C}$ and the reaction

557 time was scored when the animal began to exhibit signs of pain avoidance such as

558 jumping or paw licking. Animals that did not respond to the noxious heat stimulus after

55940 s were removed from the plate.

\section{Acetone tests}

561 For cold allodynia, $20 \mu \mathrm{L}$ acetone was applied to the ventral surface of a hind paw, and

562 then the mouse's response was observed for $60 \mathrm{~s}$. The duration of the mouse responding 563 to acetone, such as withdrawal or flick of the paw, was recorded.

565 A Rotarod system (Panlab, Spain) was used to assess motor function. Mice were tested 

accelerated from 4 to 40 rpm over a 5 min period. The falling latency was recorded.

Mice were placed in the middle of a novel open field arena $(45 \mathrm{~cm}$ length $\times 45 \mathrm{~cm}$ width

$570 \times 30 \mathrm{~cm}$ height) under normal light conditions. Using ANY-maze software (Stoelting,

SNI. The CPA protocol included pre-conditioning (baseline), conditioning, and post-

575 conditioning phases (10 min during each phase). Animals spending $>500 \mathrm{~s}$ or $<100 \mathrm{~s}$

576 of the total time in either chamber in the pre-conditioning phase were eliminated from

577 further analysis. Immediately following the pre-conditioning phase, the mice 578 underwent conditioning for $10 \mathrm{~min}$. During conditioning, one of the two chambers was 579 paired with the mechanical stimuli. The mechanical stimulus was repeated every $10 \mathrm{~s}$ 580 with a $0.16 \mathrm{~g}$ von Frey hair on the left hind paw when the mouse enters into the condition chamber. During the post-conditioning phase, the animals did not receive any stimuli and had free access to both compartments for a total of $10 \mathrm{~min}$. Animal

583 movements in each of the chambers were recorded, and the time spent in both chambers 584 was analyzed using Any-maze software. Difference scores were defined as post585 conditioning time subtracted from preconditioning time spent in the stimuli-paired chamber. 


\section{Data availability}

Sequencing data have been deposited in GEO under accession codes GSE184014. All data generated or analysed during this study are included in the manuscript and supporting file; Source Data files have been provided for Figures 1, 2, 3, 4, 6, 7, and 8,

591 and corresponding supplementary figures.

\section{Statistical analysis}

Statistical analyses were performed using GraphPad Prism (Version 8.0.1, CA, USA).

Quantitative measurements are presented as mean \pm standard errors of the means (SEM).

595 Measurements lies outside two standard deviations (SD) are excluded. Statistical

596 differences in comparison to the control group were analyzed using paired or unpaired

597 t-tests as appropriate. One-way (for multiple comparisons) or two-way ANOVA (for

598 multiple time points) with Bonferroni's post hoc tests were used for experiments with

599 more than 2 groups. Significance was considered with $p$ value $<0.05$. Regarding

600 replication, every mouse represents a replicate, and the number of replicates and

601 additional information on statistics (sample sizes, tests and $p$ values) are mentioned for 602 each experiment in the figure legend.

\section{Study approval}

604 All experiments were conducted in the Zhejiang University School of Medicine. The

605 use and care of animals in all experiments followed the guidelines of The Tab of Animal

606 Experimental Ethical Inspection of the First Affiliated Hospital, College of Medicine, 


\section{Acknowledgements}

609

610

611

612

613

614

615

616

617

618

619

620

621

We thank Dr. Zhenzhong Xu for discussions and suggestions on experimental design and technical support, Drs. Chong Liu and Liang Wang for generously providing transgenic mice, and Drs. Kaiyuan Li, Zhongya Wei, and Xiaobo Wu for the help with whole-cell patch clamp recording. We are grateful to research assistants Sanhua Fang and Daohui Zhang at the Core Facilities of Zhejiang University Institute of Neuroscience.

\section{Funding}

This work was supported by National Natural Science Foundation of China (81772382 and 32070974), National Key Research and Development Program of China (2017YFA0104200), Science Technology Department of Zhejiang Province (2020C03042), the Fundamental Research Funds for the Central Universities of China (2019FZA7009), and the Central Universities granted by Zhejiang University (No. 2021FZZX005-29).

\section{Competing interests}

The authors have declared that no conflict of interest exists.

\section{Author contributions}

Lunhao Chen: Conceptualization; Resources; Data curation; Software; Formal analysis; Investigation; Visualization; Methodology; Writing - original draft; Project administration; Writing - review and editing. Yaling Hu: Conceptualization; Data curation; Software; Formal analysis; Investigation; Methodology; Writing - original 
629 draft; Writing - review and editing. Siyuan Wang: Data curation; Investigation;

630 Methodology; Writing - original draft. Kelei Cao: Data curation; Software; Formal

631 analysis. Weihao Mai: Software. Weilin Sha: Data curation. Ma Huan: Writing - review

632 and editing. Yong-Jing Gao: Methodology; Writing - review and editing. Shumin Duan:

633 Resources; Supervision; Funding acquisition; Methodology. Yue Wang: Resources;

634 Data curation; Formal analysis; Supervision; Funding acquisition; Validation;

635 Methodology; Writing - original draft; Project administration; Writing - review and

636 editing. Zhihua Gao: Conceptualization; Data curation; Formal analysis; Supervision;

637 Funding acquisition; Validation; Investigation; Writing - original draft; Project

638 administration; Writing - review and editing. 


\section{REFERENCES}

641

642

643

644

645

646

647

648

649

650

651

652

653

654

655

656

657

658

659

660

661

662

663

664

665

666

667

668

669

670

671

672

673

674

675

676

Abdulla, F. A. and Smith, P. A. (1999a). Nerve injury increases an excitatory action of neuropeptide Y and Y2-agonists on dorsal root ganglion neurons. Neuroscience 89, 43-60. DOI: https://doi.org/10.1016/s0306-4522(98)00443-6.

Abdulla, F. A. and Smith, P. A. (1999b). Neuropeptide Y actions and the distribution of Ca2+-dependent Cl- conductance in rat dorsal root ganglion neurons. Journal of the Autonomic Nervous System 78, 249. DOI: https://doi.org/10.1016/s0165-1838(99)00058-2.

Abe, N., Borson, S. H., Gambello, M. J., Wang, F. and Cavalli, V. (2010). Mammalian target of rapamycin (mTOR) activation increases axonal growth capacity of injured peripheral nerves. Journal of Biological Chemistry 285, 28034-43. DOI: https://doi.org/10.1074/jbc.M110.125336.

Allen, Y., Adrian, T., Allen, J., Tatemoto, K., Crow, T., Bloom and Polak, J. (1983). Neuropeptide Y distribution in the rat brain. Science 221, 877-879. DOI: https://doi.org/10.1126/science.6136091.

Arcourt, A., Gorham, L., Dhandapani, R., Prato, V., Taberner, F. J., Wende, H., Gangadharan, V., Birchmeier, C., Heppenstall, P. A. and Lechner, S. G. (2017). Touch receptor-derived sensory information alleviates acute pain signaling and fine-tunes nociceptive reflex coordination. Neuron 93, 179-193. DOI: https://doi.org/10.1016/j.neuron.2016.11.027.

Asante, C. O., Wallace, V. C. and Dickenson, A. H. (2010). Mammalian target of rapamycin signaling in the spinal cord is required for neuronal plasticity and behavioral hypersensitivity associated with neuropathy in the rat. J Pain 11, 1356-67. DOI: https://doi.org/10.1016/j.jpain.2010.03.013.

Brumovsky, P., Shi, T. S., Landry, M., Villar, M. J. and Hokfelt, T. (2007). Neuropeptide tyrosine and pain. Trends in Pharmacological Sciences 28, 93-102. DOI: https://doi.org/10.1016/j.tips.2006.12.003.

Brumovsky, P., Stanic, D., Shuster, S., Herzog, H., Villar, M. and Hokfelt, T. (2005). Neuropeptide Y2 receptor protein is present in peptidergic and nonpeptidergic primary sensory neurons of the mouse. Journal of Comparative Neurology 489, 328-48. DOI: https://doi.org/10.1002/cne.20639.

Carlin, D., Golden, J. P., Mogha, A., Samineni, V. K., Monk, K. R., Gereau, R. W. T. and Cavalli, V. (2018). Deletion of Tsc2 in nociceptors reduces target innervation, ion channel expression, and sensitivity to heat. eNeuro 5. DOI: https://doi.org/10.1523/ENEURO.0436-17.2018.

Chen, W. T., Lu, N., Ding, Y., Wang, Y., Chan, L. T., Wang, X., Gao, X., Jiang, S. S. and Liu, K. (2016). Rapamycin-resistant mTOR activity is required for sensory axon regeneration induced by a conditioning lesion. eNeuro 3. DOI: https://doi.org/10.1523/Eneuro.0358-16.2016.

Colloca, L., Ludman, T., Bouhassira, D., Baron, R., Dickenson, A. H., Yarnitsky, D., Freeman, R., Truini, A., Attal, N., Finnerup, N. B., Eccleston, C., Kalso, E., Bennett, D. L., Dworkin, R. H. and Raja, S. N. (2017). Neuropathic pain. Nature Reviews Disease Primers 3, 17002. DOI: https://doi.org/10.1038/nrdp.2017.2.

Costigan, M., Scholz, J. and Woolf, C. J. (2009). Neuropathic pain: a maladaptive response of the nervous system to damage. Annual Review of Neuroscience 32, 1-32. DOI: https://doi.org/10.1146/annurev.neuro.051508.135531. 
Cui, H., Cai, F. and Belsham, D. D. (2005). Anorexigenic hormones leptin, insulin, and alphamelanocyte-stimulating hormone directly induce neurotensin (NT) gene expression in novel NTexpressing cell models. The Journal of Neuroscience 25, 9497-506. DOI: https://doi.org/10.1523/JNEUROSCI.2269-05.2005.

Decosterd, I. and Woolf, C. J. (2000). Spared nerve injury: an animal model of persistent peripheral neuropathic pain. Pain 87, 149-158. DOI: https://doi.org/10.1016/s0304-3959(00)00276-1.

Diaz-Delcastillo, M., Woldbye, D. P. D. and Heegaard, A. M. (2018). Neuropeptide Y and its involvement in chronic pain. Neuroscience 387, 162-169. DOI: https://doi.org/10.1016/j.neuroscience.2017.08.050.

Dixon, W. J. (1965). The up-and-down method for small samples. Journal of the American Statistical Association 60, 967-978. DOI: https://doi.org/Doi 10.2307/2283398.

Geppetti, P., Veldhuis, N. A., Lieu, T. and Bunnett, N. W. (2015). G Protein-Coupled Receptors: dynamic machines for signaling pain and itch. Neuron 88, 635-49. DOI: https://doi.org/10.1016/j.neuron.2015.11.001.

Geranton, S. M., Jimenez-Diaz, L., Torsney, C., Tochiki, K. K., Stuart, S. A., Leith, J. L., Lumb, B. M. and Hunt, S. P. (2009). A rapamycin-sensitive signaling pathway is essential for the full expression of persistent pain states. Journal of Neuroscience 29, 15017-15027. DOI: https://doi.org/10.1523/Jneurosci.3451-09.2009.

Gu, N., Peng, J. Y., Murugan, M., Wang, X., Eyo, U. B., Sun, D. M., Ren, Y., Dicicco-Bloom, E., Young, W., Dong, H. L. and Wu, L. J. (2016). Spinal microgliosis due to resident microglial proliferation is required for pain hypersensitivity after peripheral nerve injury. Cell Reports 16, 605-614. DOI: https://doi.org/10.1016/j.celrep.2016.06.018.

Guan, Z., Kuhn, J. A., Wang, X., Colquitt, B., Solorzano, C., Vaman, S., Guan, A. K., Evans-Reinsch, Z., Braz, J., Devor, M., Abboud-Werner, S. L., Lanier, L. L., Lomvardas, S. and Basbaum, A. I. (2016). Injured sensory neuron-derived CSF1 induces microglial proliferation and DAP12-dependent pain. Nature Neuroscience 19, 94-101. DOI: https://doi.org/10.1038/nn.4189.

Hu, Y., Mai, W., Chen, L., Cao, K., Zhang, B., Zhang, Z., Liu, Y., Lou, H., Duan, S. and Gao, Z. (2019). mTOR-mediated metabolic reprogramming shapes distinct microglia functions in response to lipopolysaccharide and ATP. Glia. DOI: https://doi.org/10.1002/glia.23760.

Inoue, K. and Tsuda, M. (2018). Microglia in neuropathic pain: cellular and molecular mechanisms and therapeutic potential. Nature Reviews: Neuroscience 19, 138-152. DOI: https://doi.org/10.1038/nrn.2018.2.

Khoutorsky, A., Bonin, R. P., Sorge, R. E., Gkogkas, C. G., Pawlowski, S. A., Jafarnejad, S. M., Pitcher, M. H., Alain, T., Perez-Sanchez, J., Salter, E. W., Martin, L., Ribeiro-Da-Silva, A., De Koninck, Y., Cervero, F., Mogil, J. S. and Sonenberg, N. (2015). Translational control of nociception via 4E-binding protein 1. eLife 4. DOI: https://doi.org/10.7554/eLife.12002.

Khoutorsky, A. and Price, T. J. (2018). Translational control mechanisms in persistent pain. Trends in Neurosciences 41, 100-114. DOI: https://doi.org/10.1016/j.tins.2017.11.006. 

93. DOI: https://doi.org/10.1016/j.cell.2012.03.017.

Laplante, M. and Sabatini, D. M. (2013). Regulation of mTORC1 and its impact on gene expression at a glance. Journal of Cell Science 126, 1713-9. DOI: https://doi.org/10.1242/jcs.125773.

Lisi, L., Aceto, P., Navarra, P. and Dello Russo, C. (2015). mTOR kinase: a possible pharmacological target in the management of chronic pain. BioMed Research International 2015, 394257. DOI: https://doi.org/10.1155/2015/394257.

Livak, K. J. and Schmittgen, T. D. (2001). Analysis of Relative Gene Expression Data Using Real-Time Quantitative PCR and the 2- $\Delta \Delta \mathrm{CT}$ Method. Methods 25, 402-408. DOI: https://doi.org/10.1006/meth.2001.1262.

Melemedjian, O. K., Asiedu, M. N., Tillu, D. V., Sanoja, R., Yan, J., Lark, A., Khoutorsky, A., Johnson, J., Peebles, K. A., Lepow, T., Sonenberg, N., Dussor, G. and Price, T. J. (2011). Targeting Adenosine Monophosphate-Activated Protein Kinase (AMPK) in preclinical models reveals a potential mechanism for the treatment of neuropathic pain. Molecular Pain 7, 1744-8069-7-70. DOI: https://doi.org/10.1186/1744-8069-7-70.

Melemedjian, O. K. and Khoutorsky, A. (2015). Translational control of chronic pain. Progress in

DOI: https://doi.org/10.1016/bs.pmbts.2014.11.006.

Melemedjian, O. K., Khoutorsky, A., Sorge, R. E., Yan, J., Asiedu, M. N., Valdez, A., Ghosh, S., Dussor, G., Mogil, J. S., Sonenberg, N. and Price, T. J. (2013). mTORC1 inhibition induces pain via IRS-1dependent feedback activation of ERK. Pain 154, 1080-91. DOI: https://doi.org/10.1016/j.pain.2013.03.021. of neuropeptide $\mathrm{Y}$ on nociceptive transmission in substantia gelatinosa of the adult rat spinal dorsal horn. Neuroscience 134, 595-604. DOI: https://doi.org/10.1016/j.neuroscience.2005.04.045.

740 Muraoka, O., Xu, B., Tsurumaki, T., Akira, S., Yamaguchi, T. and Higuchi, H. (2003). Leptin-induced transactivation of NPY gene promoter mediated by JAK1, JAK2 and STAT3 in the neural cell lines. Neurochemistry international 42, 591-601. DOI: https://doi.org/10.1016/s0197-0186(02)00160-2.

Nelson, T. S. and Taylor, B. K. (2021). Targeting spinal neuropeptide Y1 receptor-expressing interneurons to alleviate chronic pain and itch. Progress in Neurobiology 196, 101894. DOI: https://doi.org/10.1016/j.pneurobio.2020.101894.

Norsted Gregory, E., Codeluppi, S., Gregory, J. A., Steinauer, J. and Svensson, C. I. (2010). Mammalian target of rapamycin in spinal cord neurons mediates hypersensitivity induced by peripheral inflammation. Neuroscience 169, 1392-402. DOI: https://doi.org/10.1016/j.neuroscience.2010.05.067. Systemic inhibition of the mammalian target of rapamycin (mTOR) pathway reduces neuropathic pain in mice. Pain 152, 2582-95. DOI: https://doi.org/10.1016/j.pain.2011.07.025. 
D. R. and Gan, W. B. (2013). Microglia promote learning-dependent synapse formation through brainderived neurotrophic factor. Cell 155, 1596-1609. DOI: https://doi.org/10.1016/j.cell.2013.11.030.

Peng, J., Gu, N., Zhou, L., U, B. E., Murugan, M., Gan, W. B. and Wu, L. J. (2016). Microglia and monocytes synergistically promote the transition from acute to chronic pain after nerve injury. Nature Communications 7, 12029. DOI: https://doi.org/10.1038/ncomms12029.

Price, T. J. and Géranton, S. M. (2009). Translating nociceptor sensitivity: the role of axonal protein synthesis in nociceptor physiology. European Journal of Neuroscience 29, 2253-2263. DOI: https://doi.org/10.1111/j.1460-9568.2009.06786.x.

Reinhold, A. K., Batti, L., Bilbao, D., Buness, A., Rittner, H. L. and Heppenstall, P. A. (2015). Differential transcriptional profiling of damaged and intact adjacent dorsal root ganglia neurons in neuropathic pain. PLoS One 10, e0123342. DOI: https://doi.org/10.1371/journal.pone.0123342.

Risson, V., Mazelin, L., Roceri, M., Sanchez, H., Moncollin, V., Corneloup, C., Richard-Bulteau, H., Vignaud, A., Baas, D., Defour, A., Freyssenet, D., Tanti, J. F., Le-Marchand-Brustel, Y., Ferrier, B., Conjard-Duplany, A., Romanino, K., Bauche, S., Hantai, D., Mueller, M., Kozma, S. C., Thomas, G., Ruegg, M. A., Ferry, A., Pende, M., Bigard, X., Koulmann, N., Schaeffer, L. and Gangloff, Y. G. (2009). Muscle inactivation of mTOR causes metabolic and dystrophin defects leading to severe myopathy. Journal of Cell Biology 187, 859-74. DOI: https://doi.org/10.1083/jcb.200903131.

Sapunar, D., Vukojevic, K., Kostic, S. and Puljak, L. (2011). Attenuation of pain-related behavior evoked by injury through blockade of neuropeptide Y Y2 receptor. Pain 152, 1173-1181. DOI: https://doi.org/10.1016/j.pain.2011.01.045.

Saxton, R. A. and Sabatini, D. M. (2017). mTOR signaling in growth, metabolism, and disease. Cell 169, 361-371. DOI: https://doi.org/10.1016/j.cell.2017.03.035.

Solway, B., Bose, S. C., Corder, G., Donahue, R. R. and Taylor, B. K. (2011). Tonic inhibition of chronic pain by neuropeptide Y. Proceedings of the National Academy of Sciences of the United States of America 108, 7224-9. DOI: https://doi.org/10.1073/pnas.1017719108.

Taiwo, O. B. and Taylor, B. K. (2002). Antihyperalgesic effects of intrathecal neuropeptide Y during inflammation are mediated by Y1 receptors. Pain 96, 353-363. DOI: https://doi.org/10.1016/s03043959(01)00481-x.

Tateda, S., Kanno, H., Ozawa, H., Sekiguchi, A., Yahata, K., Yamaya, S. and Itoi, E. (2017). Rapamycin suppresses microglial activation and reduces the development of neuropathic pain after spinal cord injury. Journal of Orthopaedic Research 35, 93-103. DOI: https://doi.org/10.1002/jor.23328.

Tracey, D. J., Romm, M. A. and Yao, N. N. (1995). Peripheral hyperalgesia in experimental neuropathy: exacerbation by neuropeptide Y. Brain Research 669, 245-54. DOI: https://doi.org/10.1016/00068993(94)01265-J.

Van Hecke, O., Austin, S. K., Khan, R. A., Smith, B. H. and Torrance, N. (2014). Neuropathic pain in the general population: a systematic review of epidemiological studies. Pain 155, 654-62. DOI: https://doi.org/10.1016/j.pain.2013.11.013.

Wakisaka, S., Kajander, K. C. and Bennett, G. J. (1991). Increased neuropeptide Y (NPY)-like 
immunoreactivity in rat sensory neurons following peripheral axotomy. Neuroscience Letters 124, $200-$ 3. DOI: https://doi.org/10.1016/0304-3940(91)90093-9.

Wang, K., Wang, S., Chen, Y., Wu, D., Hu, X., Lu, Y., Wang, L., Bao, L., Li, C. and Zhang, X. (2021). Single-cell transcriptomic analysis of somatosensory neurons uncovers temporal development of neuropathic pain. Cell Research. DOI: https://doi.org/10.1038/s41422-021-00479-9.

Wiley, J. W., Gross, R. A. and Macdonald, R. L. (1993). Agonists for neuropeptide Y receptor subtypes NPY-1 and NPY-2 have opposite actions on rat nodose neuron calcium currents. Journal of Neurophysiology 70, 324-330. DOI: https://doi.org/10.1152/jn.1993.70.1.324.

Wu, S., Marie Lutz, B., Miao, X., Liang, L., Mo, K., Chang, Y. J., Du, P., Soteropoulos, P., Tian, B., Kaufman, A. G., Bekker, A., Hu, Y. and Tao, Y. X. (2016). Dorsal root ganglion transcriptome analysis following peripheral nerve injury in mice. Molecular Pain 12. DOI: https://doi.org/10.1177/1744806916629048.

Xia, L.-P., Luo, H., Ma, Q., Xie, Y.-K., Li, W., Hu, H. and Xu, Z.-Z. (2021). GPR151 in nociceptors modulates neuropathic pain via regulating P2X3 function and microglial activation. Brain. DOI: https://doi.org/10.1093/brain/awab245.

Xiao, H. S., Huang, Q. H., Zhang, F. X., Bao, L., Lu, Y. J., Guo, C., Yang, L., Huang, W. J., Fu, G., Xu, S. H., Cheng, X. P., Yan, Q., Zhu, Z. D., Zhang, X., Chen, Z., Han, Z. G. and Zhang, X. (2002). Identification of gene expression profile of dorsal root ganglion in the rat peripheral axotomy model of neuropathic pain. Proceedings of the National Academy of Sciences of the United States of America 99, 8360-5. DOI: https://doi.org/10.1073/pnas.122231899.

Xu, J. T., Zhao, J. Y., Zhao, X., Ligons, D., Tiwari, V., Atianjoh, F. E., Lee, C. Y., Liang, L., Zang, W., Njoku, D., Raja, S. N., Yaster, M. and Tao, Y. X. (2014). Opioid receptor-triggered spinal mTORC1 activation contributes to morphine tolerance and hyperalgesia. Journal of Clinical Investigation 124, 592603. DOI: https://doi.org/10.1172/JCI70236.

Xu, Q., Fitzsimmons, B., Steinauer, J., O'neill, A., Newton, A. C., Hua, X. Y. and Yaksh, T. L. (2011). Spinal phosphinositide 3-kinase-Akt-mammalian target of rapamycin signaling cascades in inflammation-induced hyperalgesia. Journal of Neuroscience 31, 2113-24. DOI: https://doi.org/10.1523/JNEUROSCI.2139-10.2011.

Yousuf, M. S., Shiers, S. I., Sahn, J. J., Price, T. J. and Dantzer, R. (2020). Pharmacological Manipulation of Translation as a Therapeutic Target for Chronic Pain. Pharmacological Reviews 73, 59-88. DOI: https://doi.org/10.1124/pharmrev.120.000030.

Yu, X., Liu, H., Hamel, K. A., Morvan, M. G., Yu, S., Leff, J., Guan, Z., Braz, J. M. and Basbaum, A. I. (2020). Dorsal root ganglion macrophages contribute to both the initiation and persistence of neuropathic pain. Nature Communications 11, 264. DOI: https://doi.org/10.1038/s41467-019-13839-2.

Zhang, W., Sun, X.-F., Bo, J.-H., Zhang, J., Liu, X.-J., Wu, L.-P., Ma, Z.-L. and Gu, X.-P. (2013). Activation of mTOR in the spinal cord is required for pain hypersensitivity induced by chronic constriction injury in mice. Pharmacology Biochemistry and Behavior 111, 64-70. DOI: https://doi.org/10.1016/j.pbb.2013.07.017.

Zhao, J. Y., Liang, L., Gu, X., Li, Z., Wu, S., Sun, L., Atianjoh, F. E., Feng, J., Mo, K., Jia, S., Lutz, B. 
bioRxiv preprint doi: https://doi.org/10.1101/2021.10.29.466458; this version posted November 1, 2021. The copyright holder has placed

this preprint (which was not certified by peer review) in the Public Domain. It is no longer restricted by copyright. Anyone can legally share, reuse, remix, or adapt this material for any purpose without crediting the original authors.

830 M., Bekker, A., Nestler, E. J. and Tao, Y. X. (2017). DNA methyltransferase DNMT3a contributes to

831 neuropathic pain by repressing Kcna2 in primary afferent neurons. Nature Communications 8, 14712.

832 DOI: https://doi.org/10.1038/ncomms14712.

833

834 
A

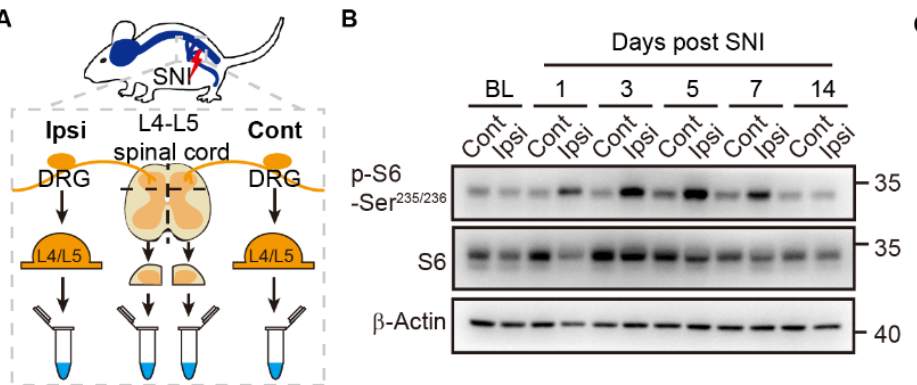

D

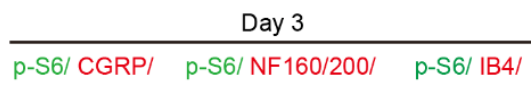

DAPI
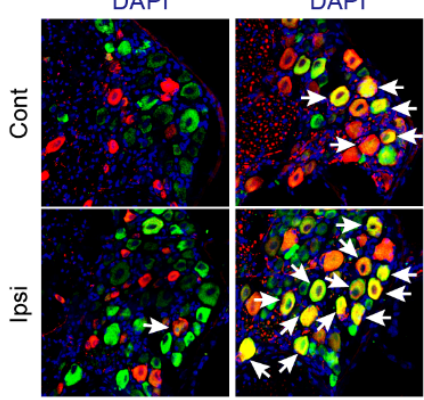

H

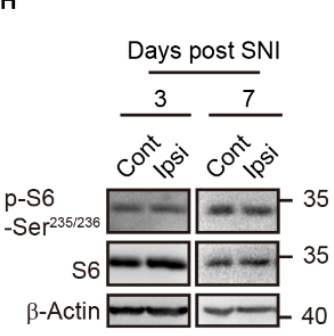

836 after spared nerve injury (SNI).

(A) A schematic diagram depicting the isolation of DRGs and SDH. ipsilateral DRGs after SNI. = 4-7 mice per time point). (arrows indicating co-labeled neurons). Scale bar, $50 \mu \mathrm{m}$. CGRP (E), NF160/200 (F), and IB4 (G) ( $n=4$ mice). SNI. 3 and day 7 post SNI respectively).
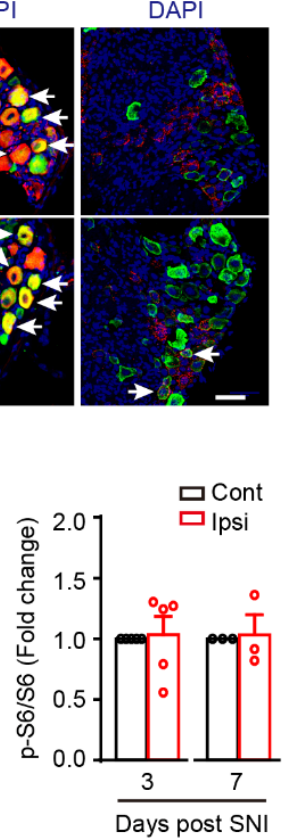

E

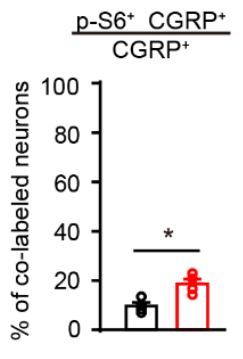

$\mathbf{F}$
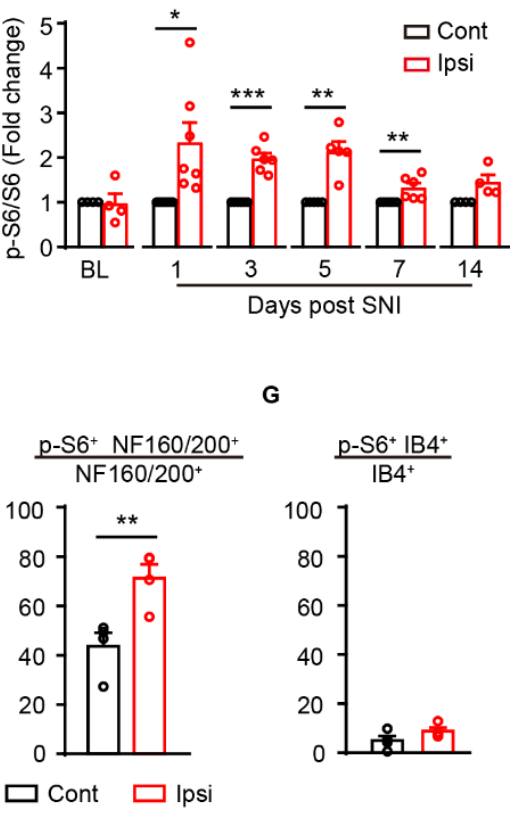

$\mathbf{J}$

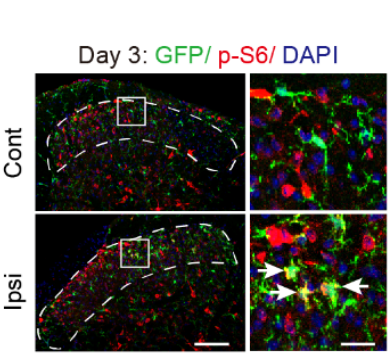

K

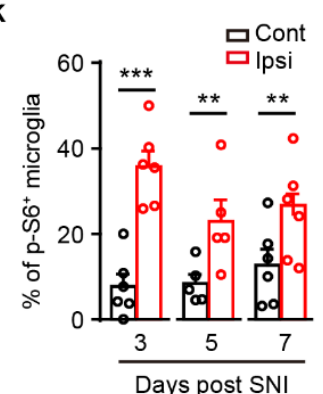

Figure 1. Activation of the mTOR in subsets of DRG neurons and SDH microglia

(B) Representative blots indicating the upregulated phosphor-S6 (p-S6) levels in the

(C) Quantification of p-S6/S6 in ipsilateral DRG at indicated time points after SNI ( $n$

(D) Co-immunostaining p-S6 with CGRP, NF160/200 or IB4 in DRGs after SNI

(E-G) Quantification of p-S6 ${ }^{+}$neurons in different subpopulations of DRG neurons:

(H) Representative blots of p-S6 and S6 levels in SDH (L4-L5) at day 3 and day 7 after

(I) Quantification of p-S6/S6 in ipsilateral and contralateral SDH ( $n=5$ and 3 for day 
852 (J) Representative images of p-S6 ${ }^{+}$microglia (arrows) in superficial contralateral and 853 ipsilateral SDH (dotted lines) at indicated time points after SNI. Boxes show regions of 854 higher magnification in the SDH. Scale bar, $100 \mu \mathrm{m}$ for low magnification images and $85520 \mu \mathrm{m}$ for high magnification images.

856 (K) Quantification of p-S6 ${ }^{+}$microglia in superficial SDH ( $n=5-6$ mice per time point).

857 Values are means \pm SEM. ${ }^{*} p<0.05$, ${ }^{* *} p<0.01$, and $* * * p<0.001$, paired student t-tests.

858 BL, baseline; Ipsi, ipsilateral; Cont, contralateral; DRG: dorsal root ganglion; SDH, 859 spinal dorsal horn.

860 Figure 1-source data 1. Raw data of quantification of p-S6/S6 blots, p-S6 ${ }^{+}$neurons, p$861 \mathrm{S6}^{+}$microglia in DRG and SDH.

862 Figure 1-source data 2. Original pictures of the western blots presented in (B) and (H).

863 Figure 1-figure supplement 1. Characterizing of p-S6 staining with different markers 864 in DRG or SDH after SNI. 
A

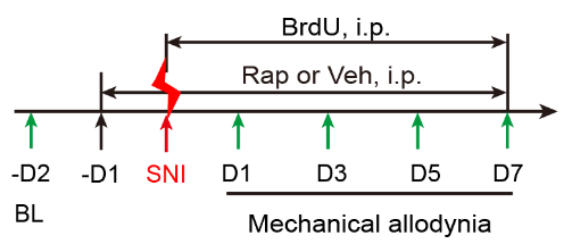

C

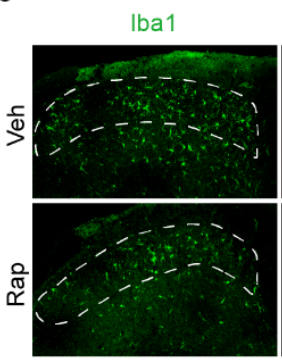

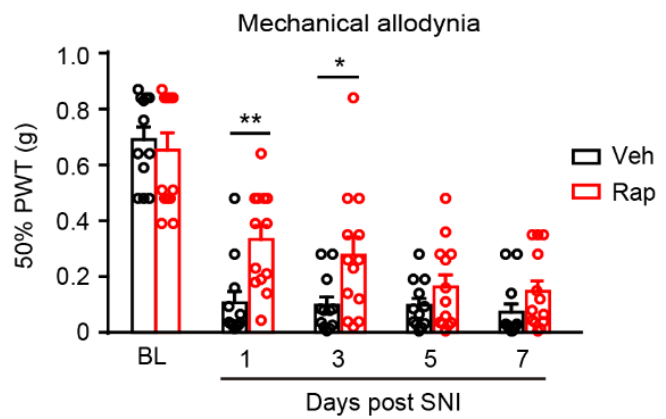

866

867

868

869

870

871

872

873

874

875

876

877

878

879

880

881

882

883

884

885

886

887

888

889

Figure 2. Rapamycin treatments inhibit mTOR activation and attenuates mechanical allodynia after SNI.

(A) Experimental schedule for rapamycin or vehicle administration through intraperitoneal (i.p.) injection.

(B) Measurements of mechanical allodynia with daily i.p. injection of rapamycin or vehicle after SNI ( $n=12-13$ per group).

(C) Representative images of Iba1 and BrdU immunolabeling in ipsilateral superficial SDH (dotted regions) at day 7 after SNI. Boxes show regions of higher magnification in $\mathrm{SDH}$, while arrows indicate $\mathrm{Iba}^{+} \mathrm{BrdU}^{+}$mitotic microglia. Scale bars, $100 \mu \mathrm{m}$ for low magnification images and $20 \mu \mathrm{m}$ for high magnification images.

(D-E) Quantitative analysis of microglia per square millimeter (D) and the percentage of mitotic microglia in total microglia (E) in ipsilateral SDH at day 7 after SNI ( $n=5$ 7 mice per group).

Values are means \pm SEM. ${ }^{*} p<0.05,{ }^{* *} p<0.01$, ${ }^{* * *} p<0.001$, two-way ANOVA followed by Bonferroni's post hoc tests among group (B), or unpaired student t-tests (D, E). Rap, rapamycin; Veh, vehicle; BL, baseline; D, day; SDH, spinal dorsal horn; PWT, paw withdraw threshold.

Figure 2-source data 1. Raw data of measurements of mechanical allodynia, number of microglia per square millimeter, and the percentage of mitotic microglia in total microglia.

Figure 2-figure supplement 1. Administration of rapamycin suppresses mTOR activation. 
A

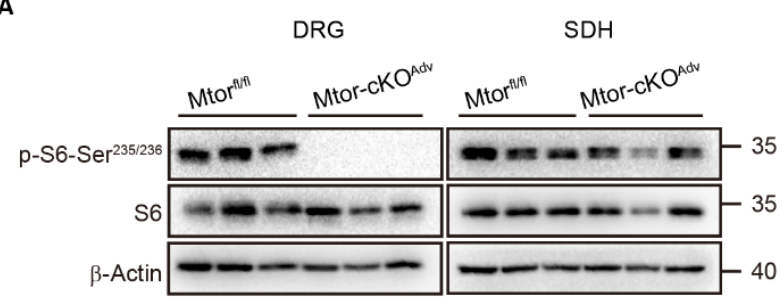

B

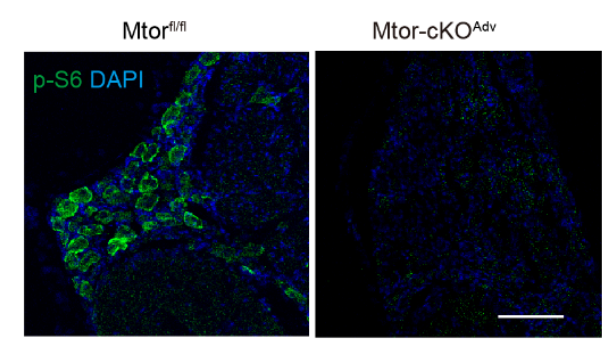

D

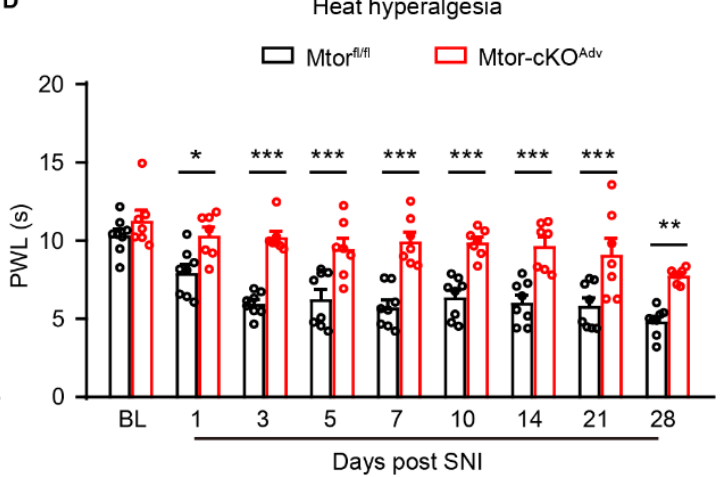

C

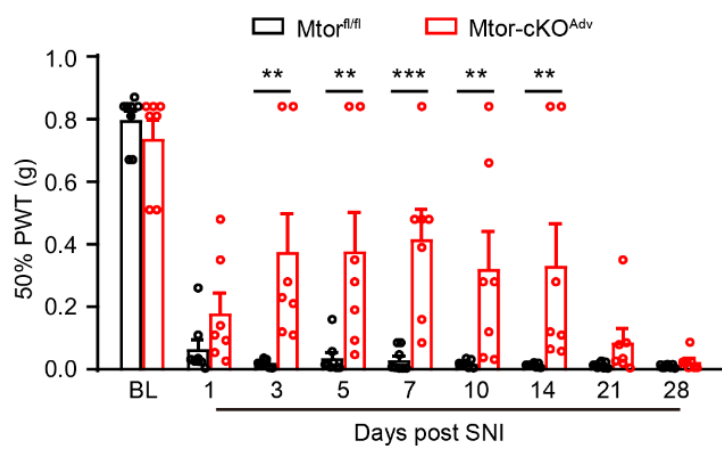

E

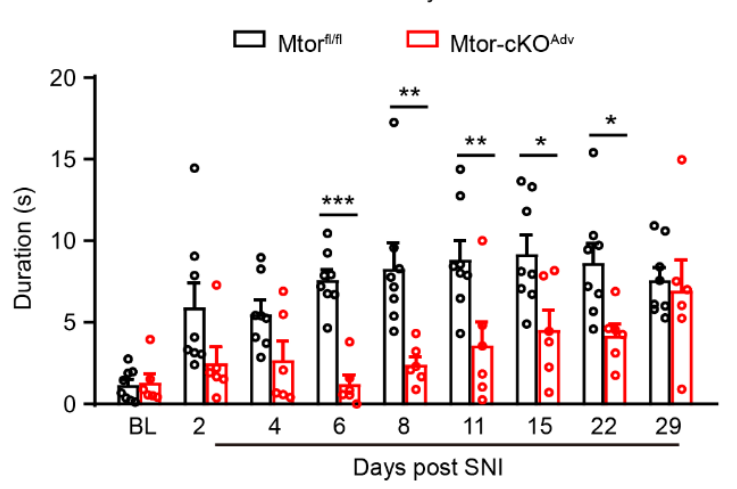

F

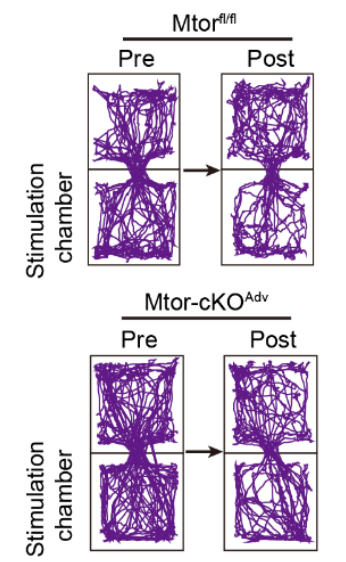

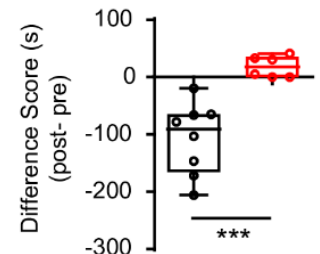

$\square$ Mtor ${ }^{\text {NIt }}$

$\square$ Mtor-cKOAdv
890

Figure 3. Ablation of Mtor in DRG neurons alleviates neuropathic pain.

(A) Representative blots of p-S6 and S6 in ipsilateral DRG and SDH from Mtor ${ }^{f l / l}$ and Mtor-cKO ${ }^{A d v}$ mice at day 7 after SNI.

(B) Representative images of p-S6 in ipsilateral DRG at day 7 after SNI, indicating the ablation of mTOR in Mtor-cKO ${ }^{A d v}$ mice rather than Mtor ${ }^{f l f l}$ mice after SNI. Scale bar, $100 \mu \mathrm{m}$.

(C-E) Measurements of mechanical allodynia (C), heat hyperalgesia (D), and cold allodynia (E) in Mtor ${ }^{f l / f l}$ and Mtor-cKO ${ }^{A d v}$ mice before and after SNI ( $n=6-8$ mice per group).

(F) Track plots of animal movements at pre- and post-conditioning phase with a twochamber conditioned place aversion (CPA) test ( $n=6-8$ mice per group) in Mtor ${ }^{f l f l}$ and Mtor-cKO ${ }^{A d v}$ mice at day 15 after SNI. Difference scores = post-conditioning time (post) - pre-conditioning (pre) time spent in the stimulation chamber.

Values are means \pm SEM. ${ }^{*} p<0.05$, $* * p<0.01, * * * p<0.001$, two-way ANOVA followed by Bonferroni's post hoc tests among groups (C-E), or unpaired student t-tests (F). BL, baseline, PWT, paw withdraw threshold; PWL, paw withdraw latency. 
907 Figure 3-source data 1. Raw data of measurements of mechanical allodynia, heat 908 hyperalgesia, cold allodynia, and CPA tests.

909 Figure 3-source data 2. Original pictures of the western blots presented in (A).

910 Figure 3-figure supplement 1. Sensory functions and motor activities are comparable 911 in Mtor ${ }^{f l / f l}$ and Mtor-cKO ${ }^{A d v}$ mice at basal state. 
A

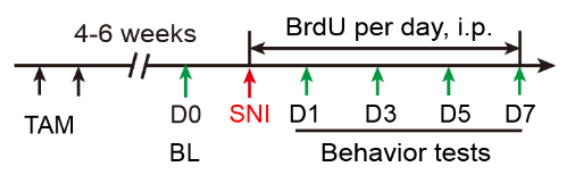

C

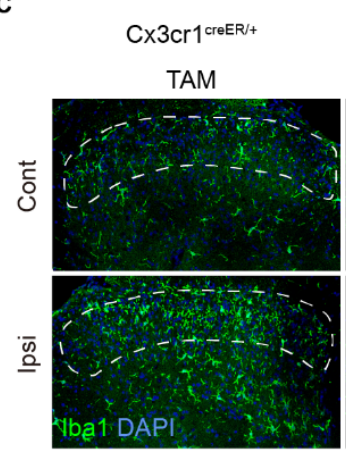

E
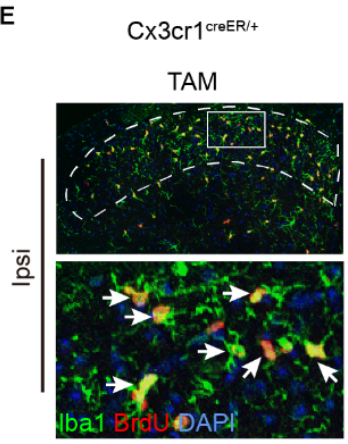

G

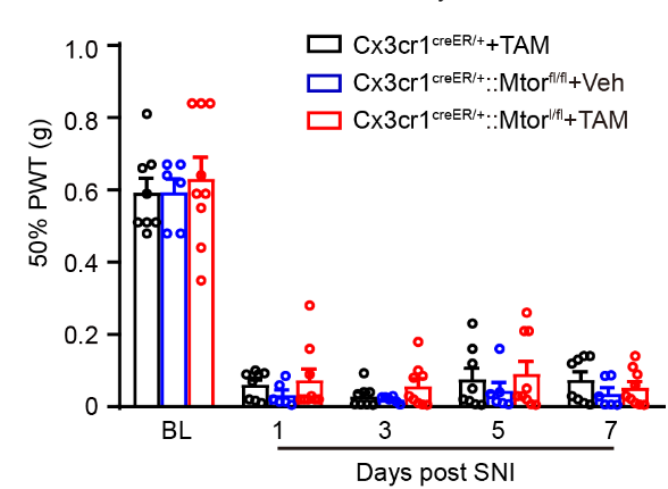

B

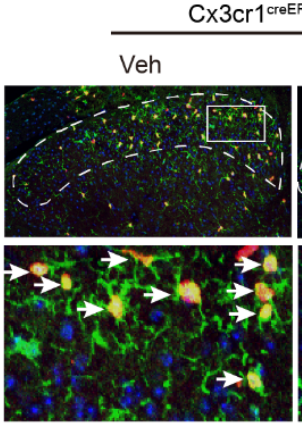

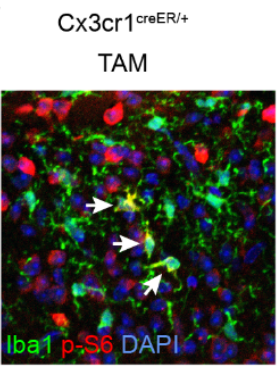

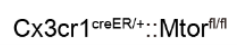
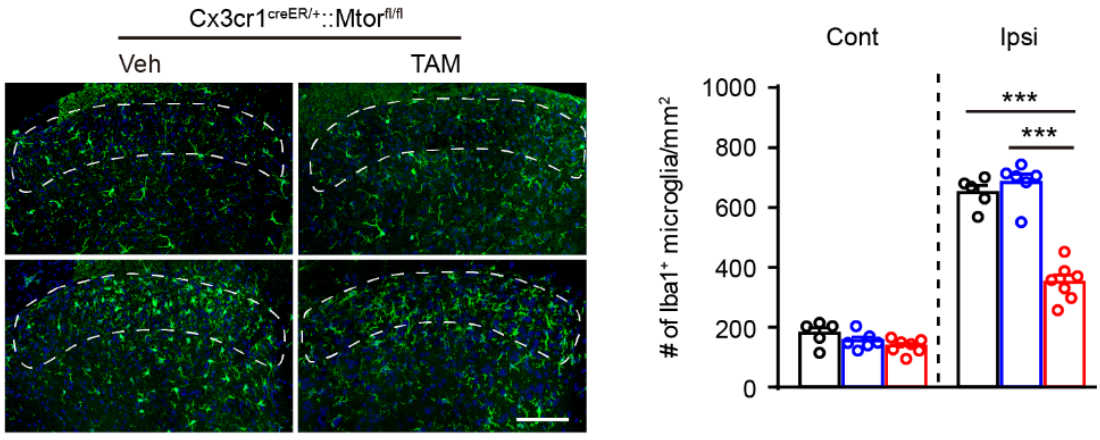

$\mathbf{F}$

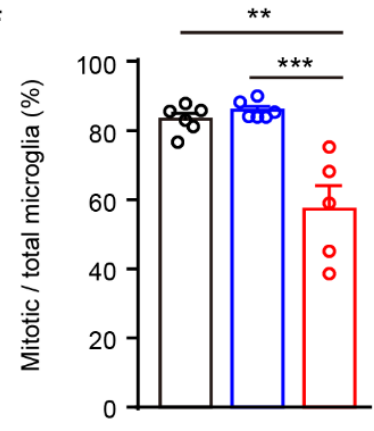

TAM
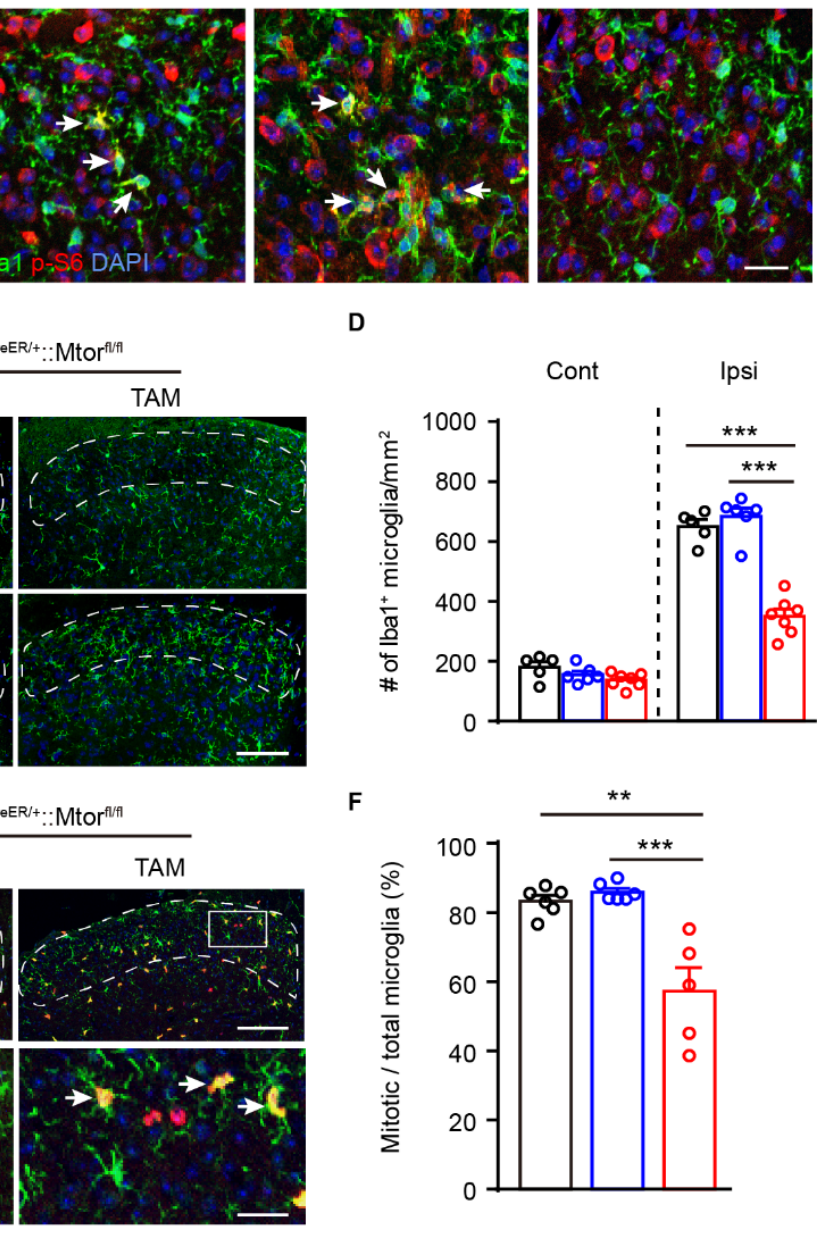

D

H

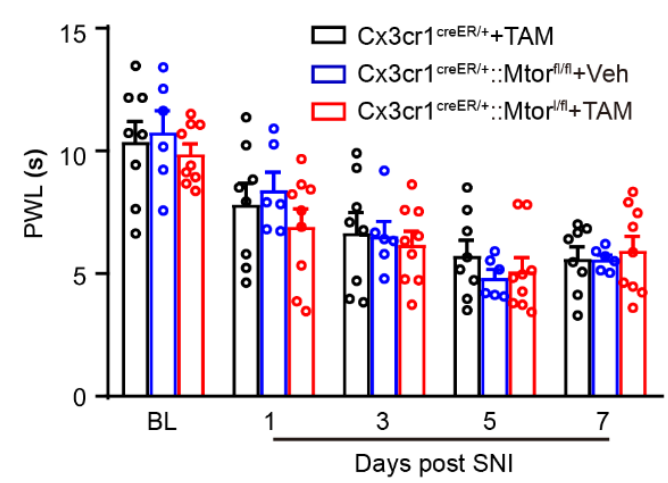

Figure 4. Ablation of Mtor in microglia reduces microgliosis, but does not affect neuropathic pain.

915 (A) Experimental schedule showing the selected Mtor deletion in microglia and pain 916 tests.

917 (B) Representative images showing immunofluorescence labeling of Iba1 and p-S6 in

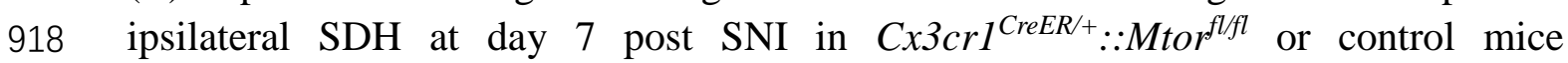
919 (Cx3cr1 $1^{C r e E R /+}$ mice with TAM and $C \times 3 c r 1^{C r e E R /+}:: M t o r^{f l / f l}$ mice with Veh). Arrows 920 indicating $\mathrm{Iba}^{+} \mathrm{p}^{-\mathrm{S} 6}{ }^{+}$microglia. Scale bar, $20 \mu \mathrm{m}$.

921 (C) Representative images of bilateral SDH microglia (Iba1 $\left.{ }^{+}\right)$in $C \times 3 c r 1^{\text {CreER/+}}::$ Mtor $^{f l f l}$ 
922 mice with TAM or in control mice at day 7 after SNI. Scale bar, $100 \mu \mathrm{m}$.

923 (D) Quantification of microglia in ipsilateral and contralateral in $C \times 3 c r 1^{\mathrm{CreER} /+}::$ Mtor $^{\mathrm{fl} / \mathrm{fl}}$

924 and control mice at day 7 post SNI ( $n=5-7$ per group).

925 (E) Representative images of ipsilateral SDH showing co-localization of Iba1 and BrdU 926 (arrows) at day 7 after SNI. Boxes show regions of higher magnification in the SDH.

927 Scale bar, $100 \mu \mathrm{m}$ for low magnification images and $20 \mu \mathrm{m}$ for high magnification 928 images.

929 (F) Quantitation of mitotic microglia $\left(\mathrm{Iba}^{+} \mathrm{BrdU}^{+}\right.$) in $\mathrm{SDH}$ in $\mathrm{Cx} 3 \mathrm{cr} 1^{\mathrm{CreER} /+}::$ Mtor $^{\mathrm{fl} / \mathrm{fl}}$ 930 and control mice at day 7 after SNI ( $n=5-7$ mice per group).

931 (G-H) Measurements of mechanical allodynia (G) and heat hyperalgesia (H) in $932 C \times 3 c r 1^{C r e E R /+}:: M t o r r^{f l / f l}$ and control mice before and after SNI ( $n=6-9$ mice per group).

933 Values are means \pm SEM. ${ }^{* *} p<0.01$, and $* * * p<0.001$, one-way AVOVA (F) or two934 way ANOVA followed by Bonferroni's post hoc tests among groups (D, G, H). TAM, 935 tamoxifen; Veh, vehicle; Cont, contralateral; Ipsi, ipsilateral; PWT, paw withdraw 936 threshold; PWL, paw withdraw latency; D, day.

937 Figure 4-source data 1. Raw data of quantification of microglia, mitotic microglia, 938 mechanical allodynia, and heat hyperalgesia.

939 Figure 4-figure supplement 1. Stratagem for generating Mtor-cKO ${ }^{M G}$ mice. 
bioRxiv preprint doi: https://doi.org/10.1101/2021.10.29.466458- this version posted November 1, 2021. The copyright holder has placed this preprint (which was not certified by peer review) in the Public Domain. It is no longer restricted by copyright. Anyone can legally share, reuse, remix, or adapt this material for any purpose without crediting the original authors.

A

Mtorntif BL Mtor ${ }^{n+17}$ D7 3413191 155

C

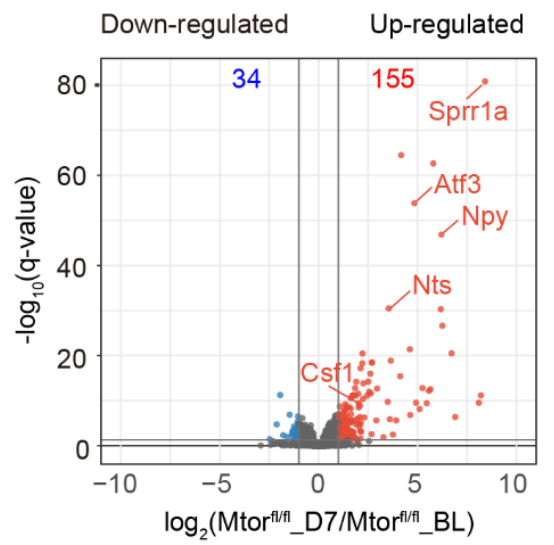

D

E

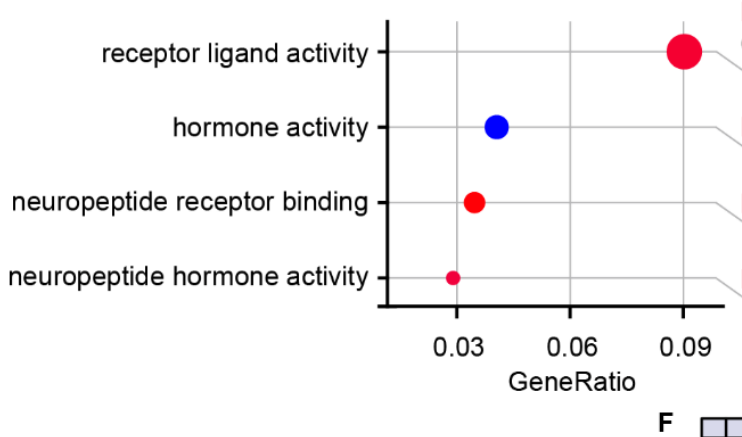

155 injury-induced genes

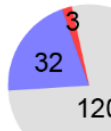

$$
\begin{aligned}
& \text { - Down-regulated } \\
& \text { - Up-regulated } \\
& \text { Unchanged }
\end{aligned}
$$

G

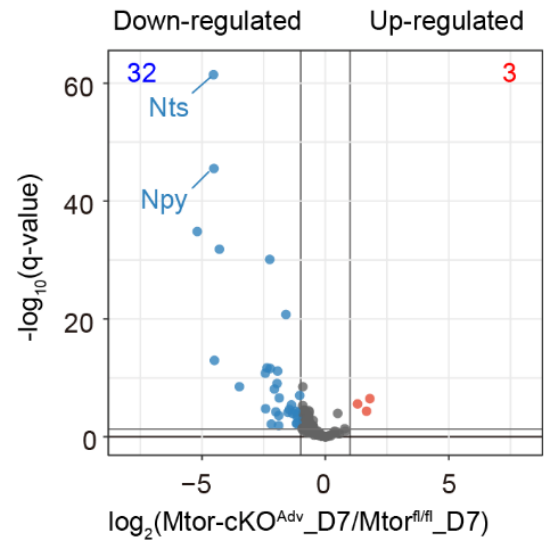

B

\section{F}

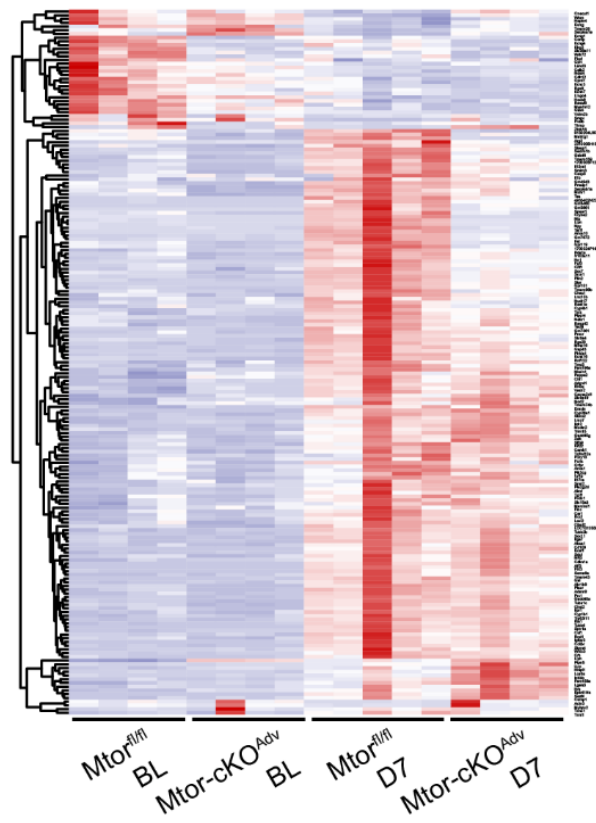

q-value

Npy/ Nts/ Crh/ Fgf3/ Gal/ Flrt3/ Sectm1b/ Crlf1/ Csf1/ II b/ Inhbb/ Lgals3/ Ucn

Npy/ Nts/ Gal/ Crh/ Ucn/ Inhbb

Npy/ Gal/ Crh/ Ucn/ Cckbr

Npy/ Nts/ Gal/ Crh

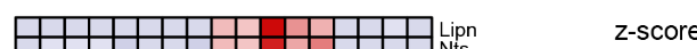

z-score

941 Figure 5. Ablation of Mtor in DRG neurons suppressed elevation of nerve injury942 induced genes.

943 (A) Venn diagram of DEGs identified in DRG before and after SNI (day 7) in Mtor fl/fl $^{\text {(d) }}$ 944 mice (155 upregulated and 34 downregulated). 
945 (B) Heat map of 189 DEGs by hierarchical clustering using z-score values $(n=4-5$ 946 mice per group).

947 (C) Volcano plot of DRG transcripts before and after SNI (day 7) in Mtor ${ }^{\text {fl/fl }}$ mice. Red 948 dots indicate 155 upregulated genes and blue dots indicate 34 downregulated genes 949 after SNI.

950 (D) GO analysis of 155 upregulated genes after SNI and regroup into molecular 951 function terms. All genes in each term are listed.

952 (E) Pie chart of 155 injury-induced genes with 32 downregulated and 3 upregulated in 953 Mtor-cKO ${ }^{\text {Adv }}$ mice after SNI.

954 (F) Heat map of 35 DEGs in all samples using Z-score values. Only 3 (Inhbb, Lce6a 955 and Ucn) of the 155 injury-induced genes are upregulated upon deletion of Mtor in 956 DRG neurons.

957 (G) Volcano plot of 35 DEGs in control and Mtor-cKO ${ }^{A d v}$ mice after SNI. Red dots 958 indicate 3 upregulated genes and blue dots indicate 32 downregulated genes after 959 mTOR ablation.

960 BL, baseline; D, day; DEGs, differentially expressed genes.

961 Figure 5-source data 1. FPKMs of differentially expressed genes (189 genes) used to 962 generate Figure B.

963 Figure 5- source data 2. FPKMs of differentially expressed genes (35 genes) upon 964 Mtor ablation used to generate Figure F.

965 Figure 5-figure supplement 1. Quantitative RT-PCR of downregulated DEGs 966 identified in RNA sequencing. 
A

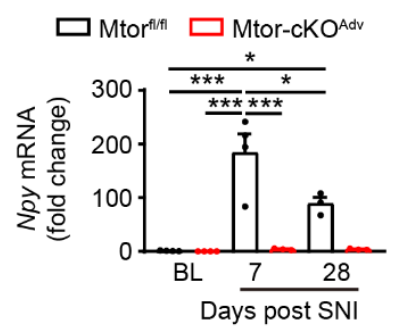

B
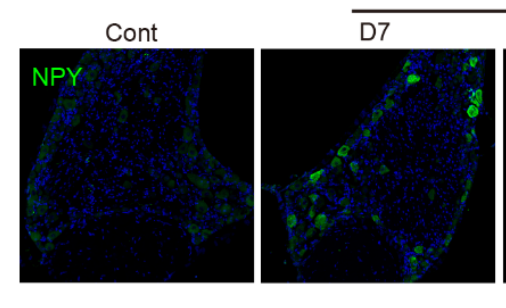

Magnified

Merge

D

C
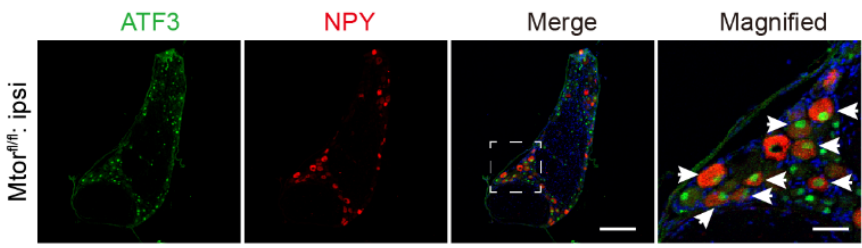

Ipsi
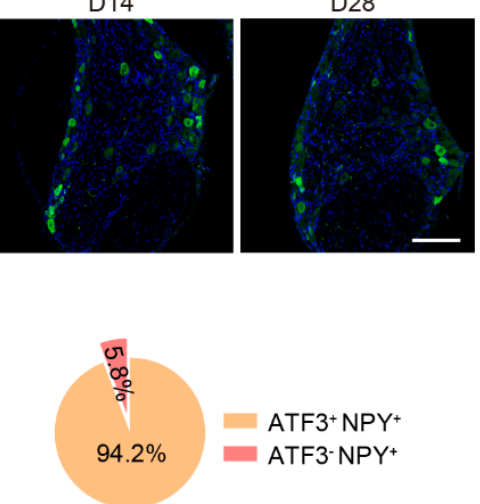

E

p-S6

NPY

Merge
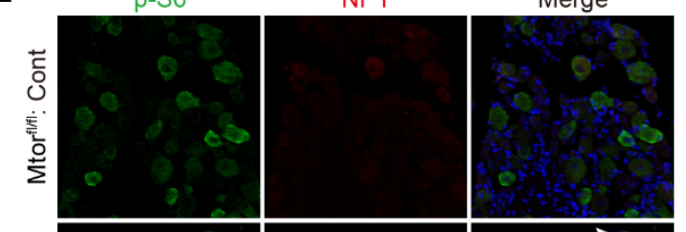

H p-S6

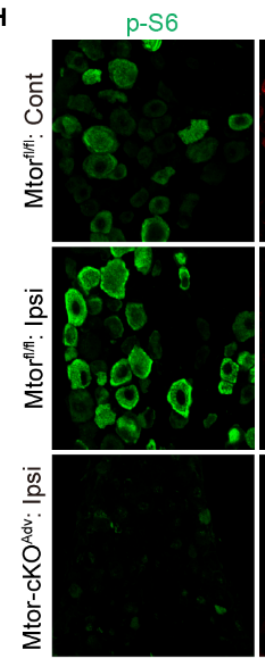

p-STAT3
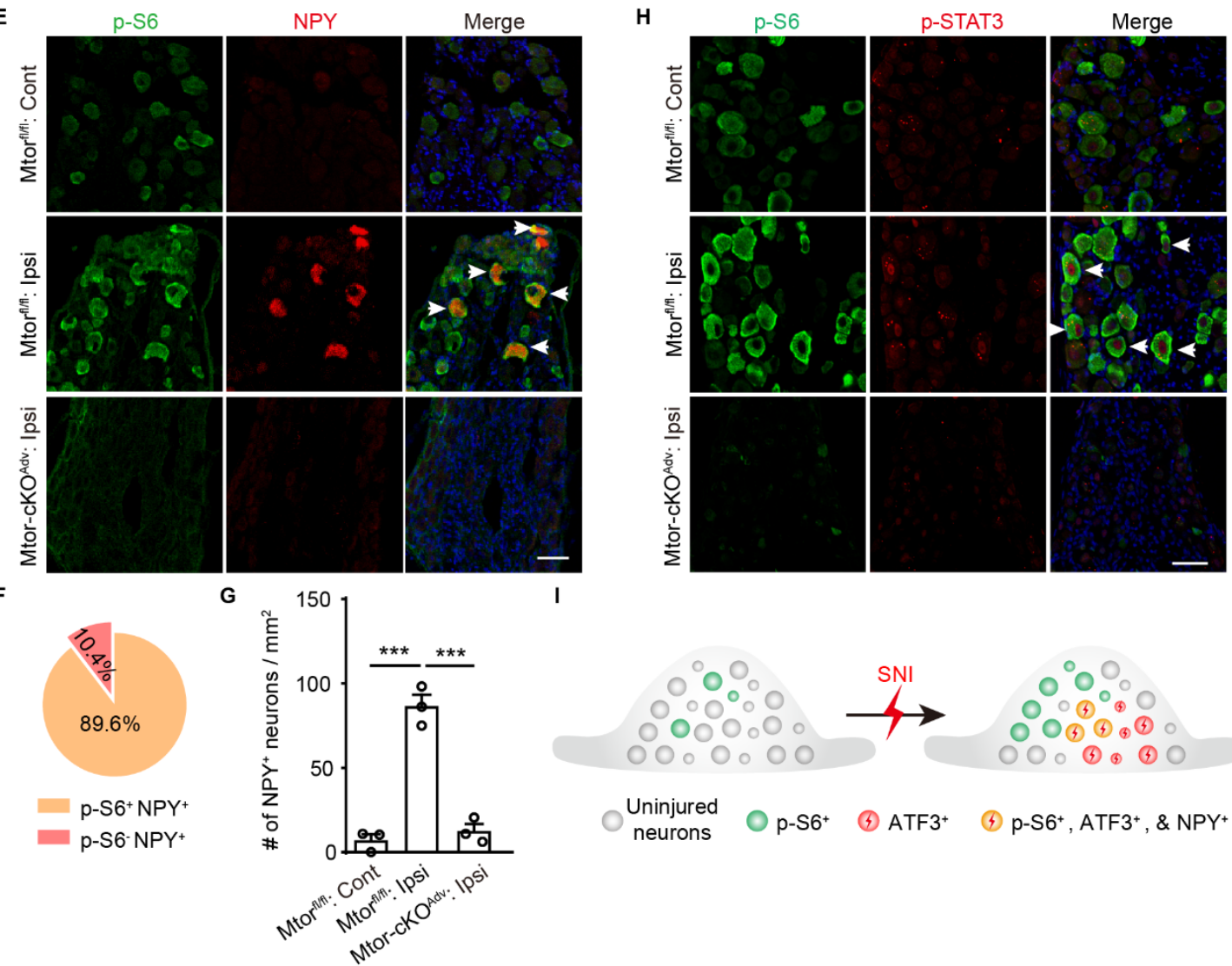

967

968

Figure 6. Activation of mTOR is required for NPY induction in DRG neurons after SNI.

(A) Quantitative RT-PCR of Npy transcripts in the ipsilateral DRG from both Mtor fl/fl and Mtor-cKO ${ }^{A d v}$ mice at indicated time points after SNI ( $n=3-4$ mice per time point).

(B) Representative images of NPY staining in DRG from Mtor flfl mice at indicated times. Scale bars, $100 \mu \mathrm{m}$.

(C) Representative images of ATF3 and NPY staining in the ipsilateral DRG from Mtor $^{\text {fl/fl }}$ mice at day 7 after SNI. Arrows indicate $\mathrm{ATF}^{+} \mathrm{NPY}^{+}$neurons. Dotted boxes show regions of higher magnification in the DRG. Scale bars, $200 \mu \mathrm{m}$ for low magnification images and $50 \mu \mathrm{m}$ for high magnification images.

(D) A pie chart showing the ratio of $\mathrm{NPY}^{+}$in total $\mathrm{ATF}^{+}$neurons in the ipsilateral DRG 
979 from Mtor $^{f l / f l}$ mice at day 7 after SNI.

980 (E) Representative images of NPY and p-S6 staining in contralateral or ipsilateral DRG 981 at day 7 after SNI in Mtor fl/fl and Mtor-cKO ${ }^{A d v}$ mice. Arrows indicate co-labeled neurons. 982 Dotted boxes show regions of higher magnification in the DRG. Scale bar, $50 \mu \mathrm{m}$.

983 (F) A pie chart indicating the ratio of $\mathrm{NPY}^{+}$neurons in all $\mathrm{p}^{-\mathrm{S}^{+}}{ }^{+}$neurons in ipsilateral 984 DRG from Mtor $^{f l / f l}$ mice at day 7 after SNI.

985 (G) Quantification of $\mathrm{NPY}^{+}$neurons in Mtor ${ }^{f l f l}$ and Mtor-cKO ${ }^{A d v}$ mice at day 7 after 986 SNI ( $n=3$ mice per group).

987 (H) Representative images of p-S6 and p-STAT3 staining in contralateral or ipsilateral 988 DRG at day 3 after SNI in $m T O R^{f l f l}$ and Mtor-cKO ${ }^{A d v}$ mice. Scale bar, $50 \mu \mathrm{m}$.

989 (I) Schematic diagram demonstrating that NPY is selectively induced in p-S6 ${ }^{+}$and $990 \mathrm{ATF3}^{+}$injured neurons in ipsilateral DRG.

991 Values are means \pm SEM. $* p<0.05$, and $* * * p<0.001$, one-way ANOVA followed by 992 Bonferroni's post hoc tests among groups. Cont, contralateral; Ipsi, ipsilateral.

993 Figure 6-source data 1. Raw data of Npy transcripts and number of $\mathrm{NPY}^{+}$neurons.

994 Figure 6-figure supplement 1. NPY was expressed in large-sized mechanoreceptors. 
A

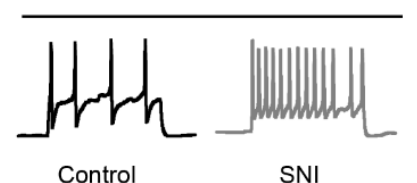

B
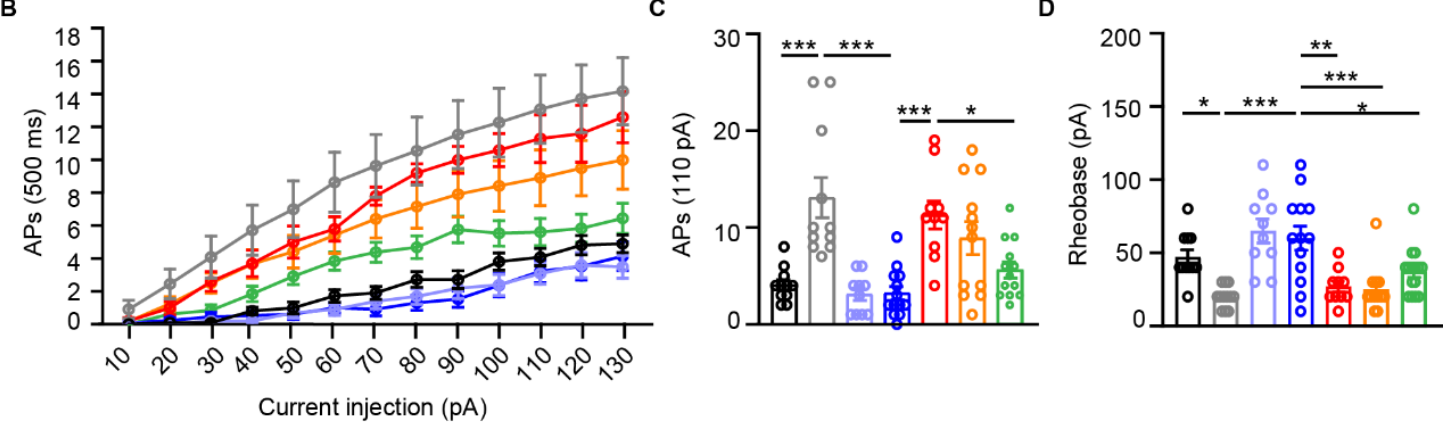

E

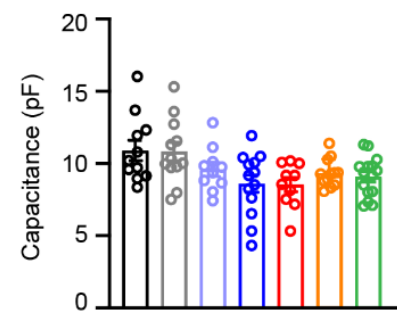

$\mathbf{F}$
996

Figure 7. NPY enhances nociceptor excitability through Y2R.

(A) Representative AP traces elicited by intracellular injection of 110 pA depolarizing currents on dissociated DRG neurons from resting membrane potentials (RMP) in Mtor ${ }^{\text {fl/fl }}$ mice and Mtor-cKO ${ }^{A d v}$ mice with or without SNI. NPY (300 nM), BIBO3304 $(1 \mu \mathrm{M})$ and BIIE0246 $(1 \mu \mathrm{M})$ are replenished in medium as indicated.

(B) The response of Mtorflfl and Mtor-cKO ${ }^{A d v}$ DRG neurons across a series of $500 \mathrm{~ms}$ depolarizing current pulses in $10 \mathrm{pA}$ increment from $0 \mathrm{pA}$ to $130 \mathrm{pA}$, in the presence or absence of NPY, BIBO3304 or BIIE0246 ( $n=10-13$ neurons per group).

(C) Quantification of APs evoked by input current at $110 \mathrm{pA}$ ( $n=10-13$ neurons per group).

(D) Averaged values of rheobase currents in DRG neurons among groups measured in I-clamp ( $n=10-13$ neurons per group).

(E-F) Quantification of membrane capacitance (E) and RMP (F) among groups $(n=$ 10-13 neurons per group). BIBO3304, Y1R antagonist; BIIE0246, Y2R antagonist.

Values are means \pm SEM. ${ }^{*} p<0.05,{ }^{* *} p<0.01$, and $* * * p<0.001$, one-way ANOVA followed by Bonferroni's post hoc tests among groups. AP, action potential; RMP, resting membrane potentials.

Figure 7-source data 1. Raw data of APs, rheobase currents, membrane capacitance, and RMP.

Figure 7-figure supplement 1. Distinct expression pattern of NPY $\left(^{*}\right)$ and Y2R (arrows) by immunofluorescence analysis. 
A

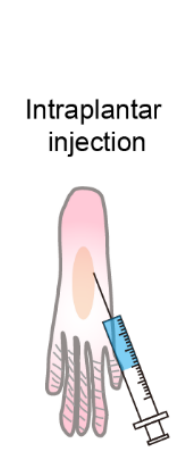

B

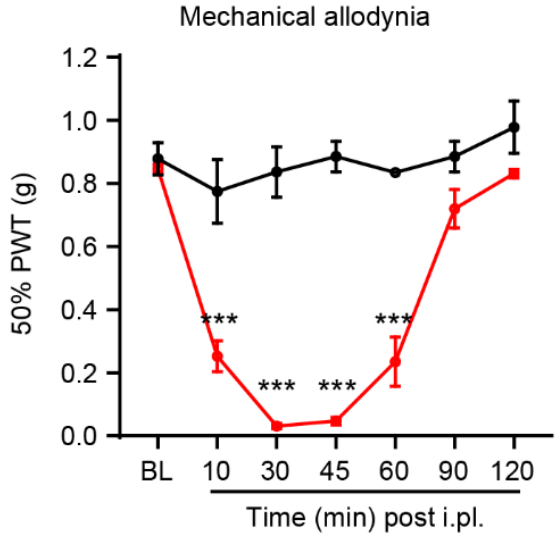

C

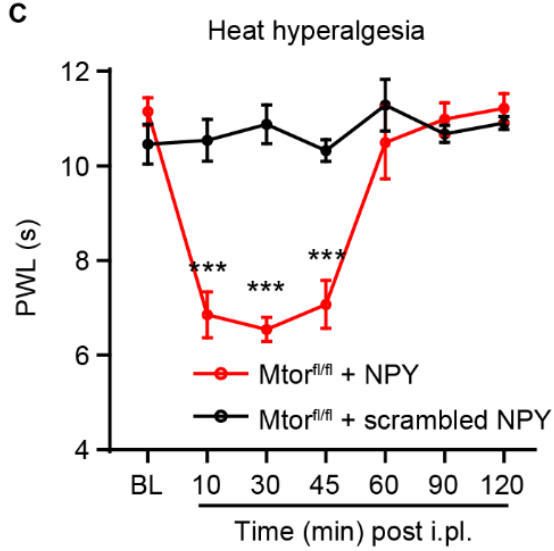

D

\begin{tabular}{|c|c|c|c|c|c|c|c|}
\hline & 4 & & $\downarrow$ & $\downarrow$ & $\downarrow$ & & Drugs, i,pl. \\
\hline $\bar{\uparrow}$ & $\uparrow$ & $\uparrow$ & $\bar{\uparrow}$ & $\bar{\uparrow}$ & $\bar{\uparrow}$ & $\bar{\uparrow}$ & $\overrightarrow{\text { Behavior tests }}$ \\
\hline$B L$ & SNI & 3 & 4 & 6 & 8 & 9 & \\
\hline
\end{tabular}

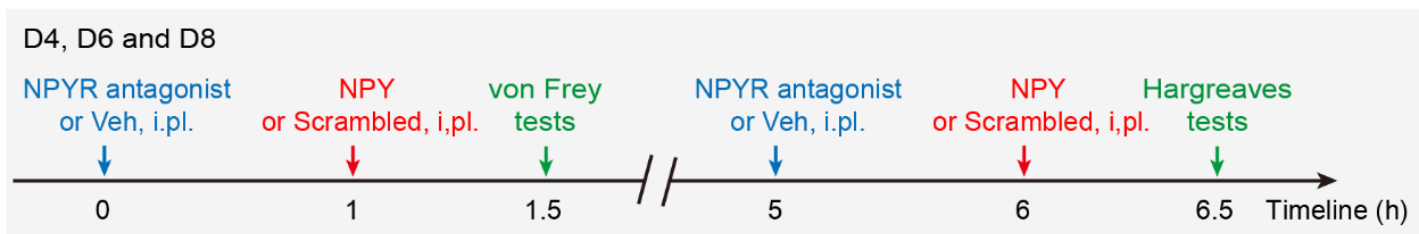

E

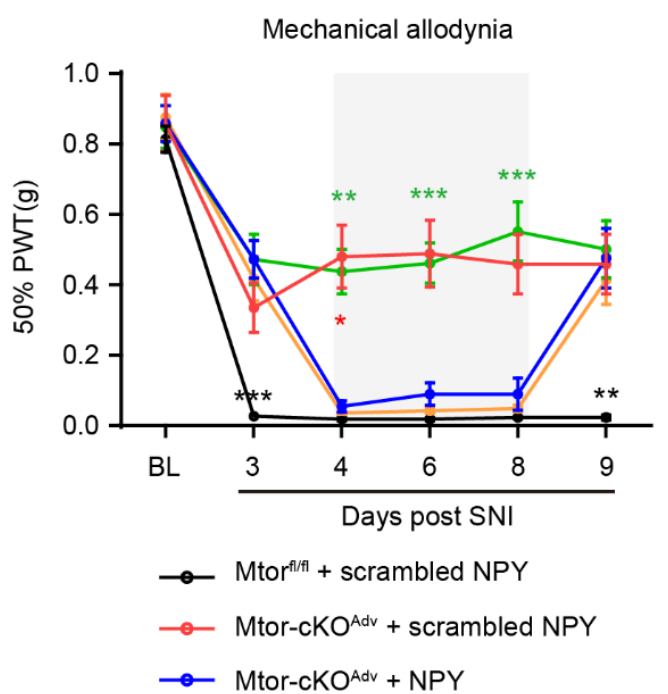

F

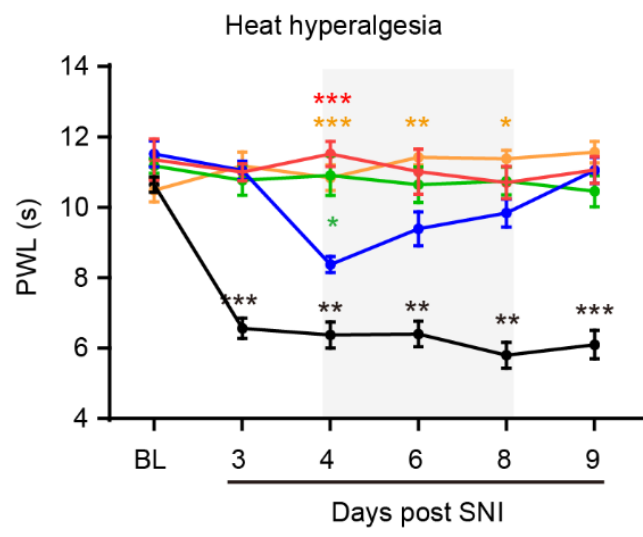

Figure 8. Intraplantar injection of NPY reversed analgesic effects of Mtor ablation through Y2R.

(A) Schematic diagram indicating intraplantar (i.pl.) injection.

(B-C) NPY (0.2 nmol) i.pl. injection into normal Mtor fl/fl mice hind paw leads to transient mechanical allodynia $(\mathbf{B})$ and heat hyperalgesia $(\mathbf{C})$ within an hour $(n=4$ mice per group).

(D) Experimental schedule showing the timeline of i.pl. injection of drugs (including NPY, NPYR antagonist and vehicle) and behavior tests. Behavior tests were measured before and after SNI as indicated. Drugs were injected at day 4, 6 and 8. 
1029 and Mtor-cKOAdv mice with i.pl. injection with NPY (0.2 nmol), scrambled NPY (0.2

$1030 \mathrm{nmol})$, BIBO3304 (5 nmol) or BIIE0246 (50 nmol) at day 4, 6, and 8 after SNI ( $\mathrm{n}=6$ 103111 mice per group). BIBO3304, Y1R antagonist; BIIE0246, Y2R antagonist.

1032 Values are means \pm SEM. * $p<0.05$, ** $p<0.01$, and *** $p<0.001$ vs. Mtor-cKO ${ }^{\text {Adv }}$ with 1033 NPY, two-way ANOVA followed by Bonferroni's post hoc tests among groups. BL, 1034 baseline; i.pl., intraplantar; Veh, vehicle; PWT, paw withdraw threshold; PWL, paw 1035 withdraw latency.

1036 Figure 8-source data 1. Raw data of mechanical allodynia and heat hyperalgesia. 
Table 1. Primer sequences in RT-PCR.

\begin{tabular}{lll}
\hline Gene name & Forward & Reverse \\
\hline Nts & CCTGACTCTCCTGGCTTTCA & CCGGGCTGTTCACGTTATTT \\
Npy & GGACTGACCCTCGCTCTATC & CTTCAAGCCTTGTTCTGGGG \\
Annexin a10 & CATCCTAACACAACGCAGCA & AGTTCCTGGTCCCTTCATGG \\
Lipn & AAGTTCGGAAGTCCTCTGGG & GAATCCACCAGCGCTTAAGG \\
Gpr119 & GTCACTATCAGCCATCCGGA & GCTGGCCGACTTCTAGAGAT \\
Fst & CGAATGTGCACTCCTCAAGG & ACTGTTCAGAAGAGGAGGGC \\
Gpr151 & GTATGGCATGTGAAGGCTGG & GCCTCCTGAACCTCTGAAGT \\
Fgf3 & ACCTGGCCATGAACAAGAGA & ACACGTACCAAGGTCTCTGG \\
Csf1 & TGCTAAGTGCTCTAGCCGAG & CCCCCAACAGTCAGCAAGAC \\
Actin & GTGACGTTGACATCCGTAAAGA & GCCGGACTCATCGTACTCC \\
\hline
\end{tabular}




\section{Supplementary material}

A

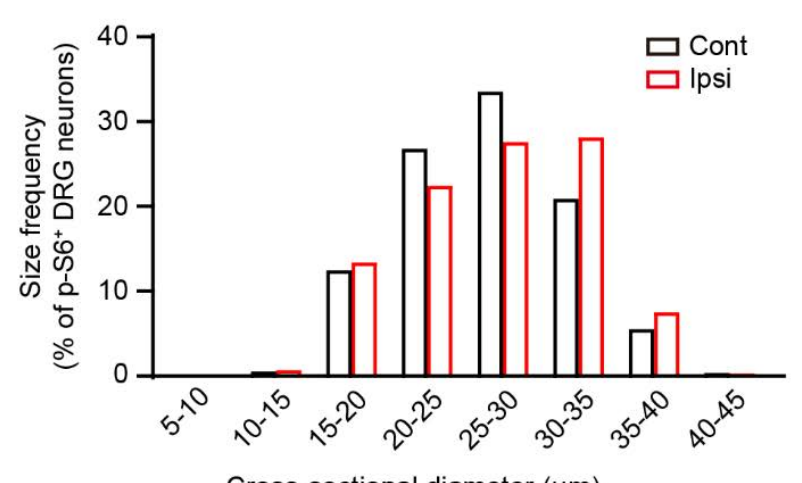

Cross-sectional diameter $(\mu \mathrm{m})$

C
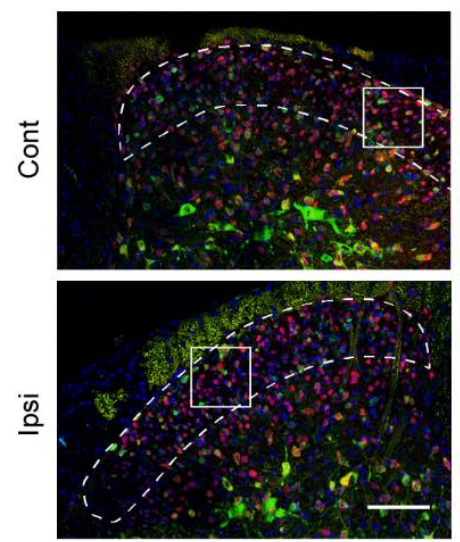

B

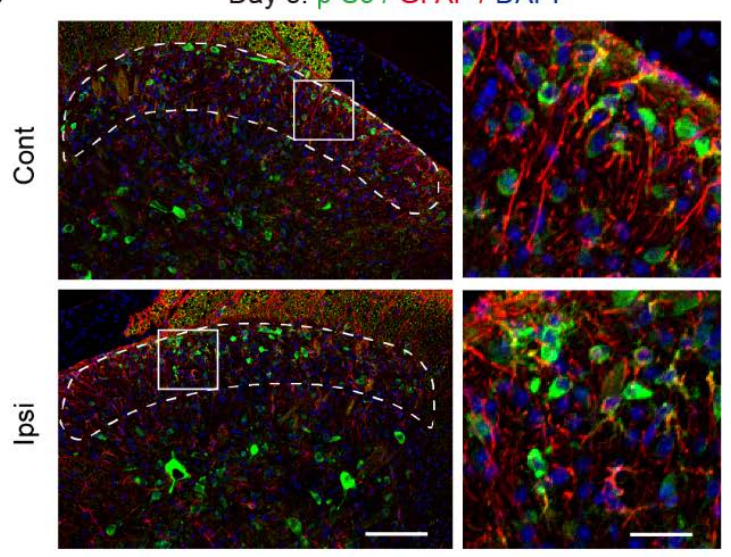

D

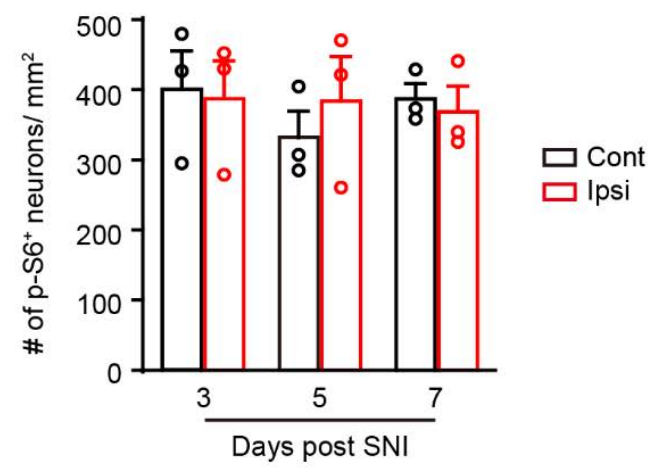

Figure 1-figure supplement 1. Characterizing of p-S6 staining with different markers in DRG or SDH after SNI.

1044 (A) Size frequency distribution patterns of p-S6 positive neurons from contralateral and ipsilateral DRG at day 3 post SNI (n=579 and 718 neurons from 3-4 mice respectively).

(B) Representative images of p-S6 and GFAP in superficial SDH (dotted regions) at day 5 after SNI. Boxes show the region with magnification.

1048 (C) Representative images of p-S6 and NeuN immunolabeling (arrows) in superficial 1049 SDH (dotted regions) at day 5 after SNI. Boxes show the region with magnification. 1050 Scale bars, $100 \mu \mathrm{m}$ and $20 \mu \mathrm{m}$ for lower- and higher-magnification images, respectively. (D) Quantitation of p-S6 ${ }^{+}$neurons in superficial SDH at day 3 to 7 after SNI ( $\mathrm{n}=3$ mice 1052 per time point). Values are means \pm SEM. Two-way ANOVA followed by Bonferroni’s 
bioRxiv preprint doi: https://doi.org/10.1101/2021.10.29.466458; this version posted November 1, 2021. The copyright holder has placed

this preprint (which was not certified by peer review) in the Public Domain. It is no longer restricted by copyright. Anyone can legally share, reuse, remix, or adapt this material for any purpose without crediting the original authors.

1053 post hoc tests among groups. Scale bars, $100 \mu \mathrm{m}$ and $20 \mu \mathrm{m}$ for lower- and higher-

1054 magnification images, respectively. Ipsi, ipsilateral; Cont, contralateral.

1055 Figure 1-figure supplement 1-source data 1. Source data used to generate Figure A 1056 and D. 
A

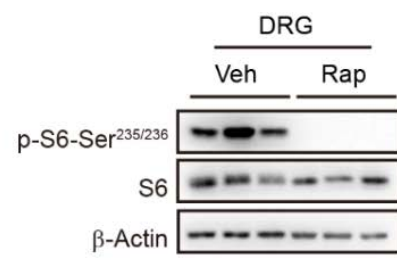

c

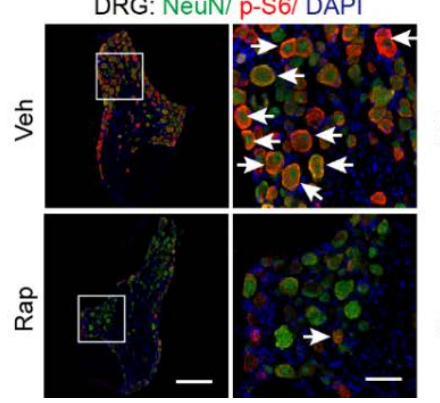

D

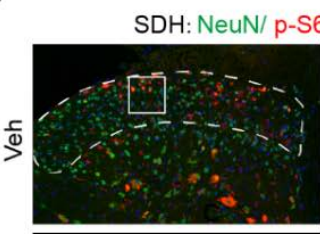

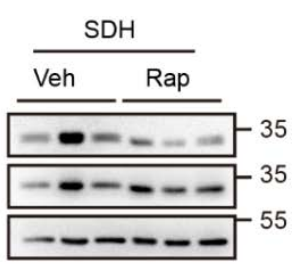

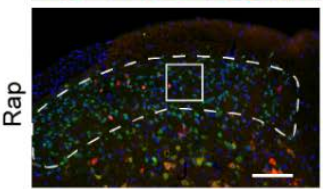

B

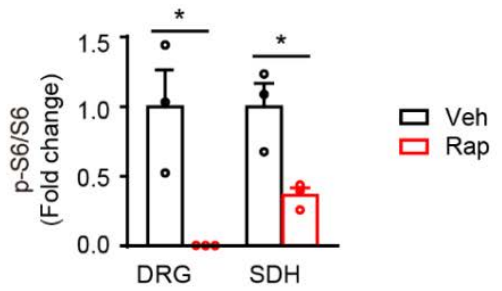

E

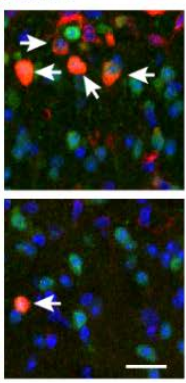

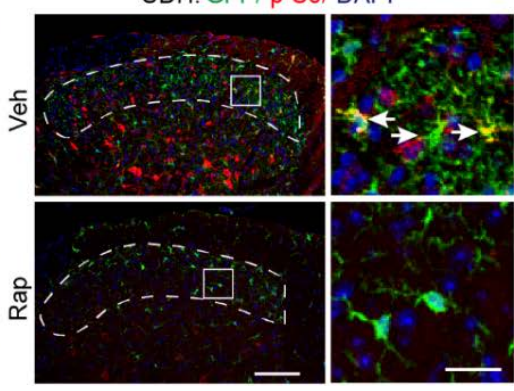

1059

1060

1061

1062

1063

1064

1065

1066

1067

1068

1069

1070

1071

1072

1073

1074

1075

Figure 2-figure supplement 1. Administration of rapamycin suppresses mTOR activation.

(A) Representative blots indicating the decreased p-S6 levels in the ipsilateral DRG and SDH following 7-day continuous intraperitoneal (i.p.) injection of rapamycin or vehicle in Mtor ${ }^{f l / f l}$ mice.

(B) Quantitation of p-S6/S6 in DRG and SDH following rapamycin treatments ( $\mathrm{n}=3$ mice per group).

(C-E) Co-immunostaining of p-S6 with NeuN or GFP in ipsilateral DRG (C) and SDH (D, E) after i.p. administration of vehicle or rapamycin (arrows indicating p-S6 ${ }^{+}$cells) at day 7 after SNI. Boxes show regions with magnification. Scale bars, $100 \mu \mathrm{m}$ and 20 $\mu \mathrm{m}$ in (C), and $200 \mu \mathrm{m}$ and $50 \mu \mathrm{m}$ in (D, E) for lower- and higher-magnification images, respectively.

Values are means \pm SEM. ${ }^{*} p<0.05$ and ${ }^{* *} p<0.01$ versus Veh, unpaired student t-tests (B). Rap, rapamycin; Veh, vehicle; BL, baseline; SDH, spinal dorsal horn.

Figure 2-figure supplement 1-source data 1. Source data used to generate Figure B.

Figure 2-figure supplement 1-source data 2. Original pictures of the western blots presented in Figure A. 
A

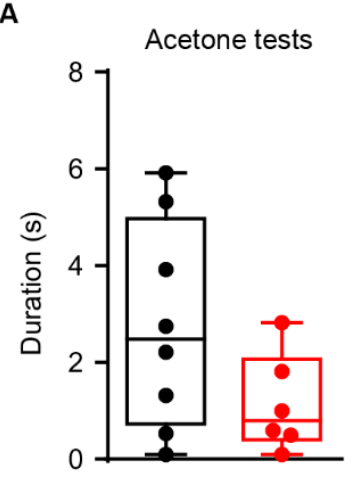

D

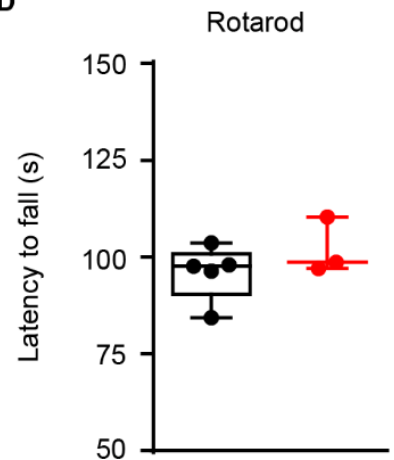

B

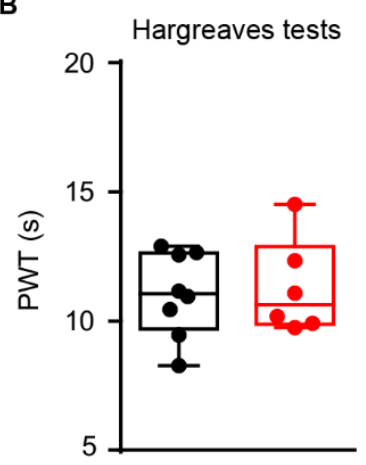

E

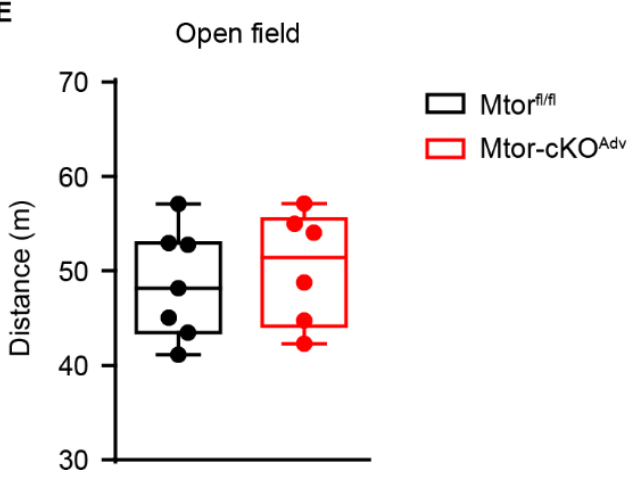

C

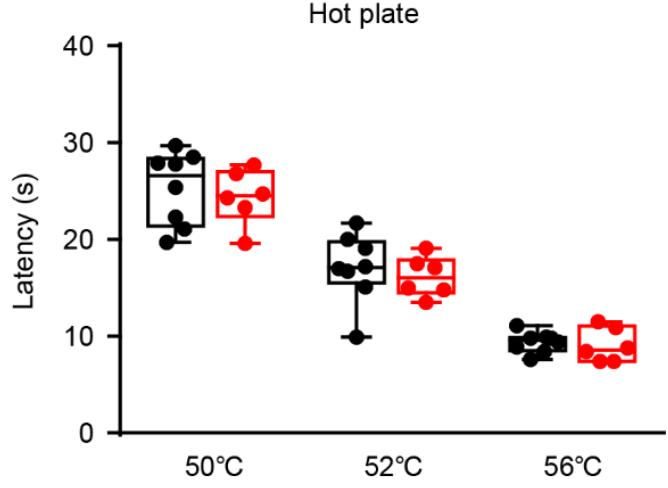

1077

1078

Figure 3-figure supplement 1. Sensory functions and motor activities are comparable in Mtor $^{f l / f l}$ and $M t o r-c K O^{A d v}$ mice at basal state.

1080 (A) Acetone tests ( $\mathrm{n}=6-8$ mice per group).

1081

(B) Hargreaves tests ( $\mathrm{n}=6-8$ mice per group).

1082

(C) Hot plate tests ( $\mathrm{n}=6-8$ mice per group).

1083

(D) Rotarod tests ( $\mathrm{n}=3-5$ mice per group).

1084

(E) Open field tests in Mtor ${ }^{f l / f l}$ and $M t o r-c K O^{A d v}$ mice (n=6-7 mice per group).

1085

Values are means \pm SEM. Unpaired student t-tests $(\mathbf{A}, \mathbf{B}, \mathbf{D}, \mathbf{E})$ and two-way ANOVA

followed by Bonferroni's post hoc tests among groups (C).

1087

Figure 3-figure supplement 1-source data 1. Source data used to generate Figure A-

1088

E. 


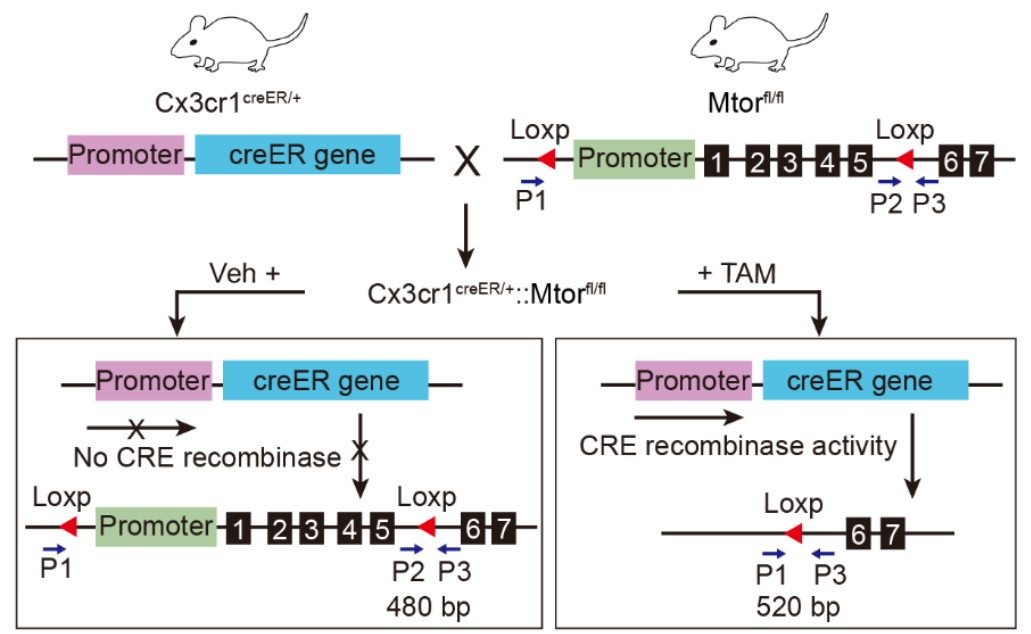

B

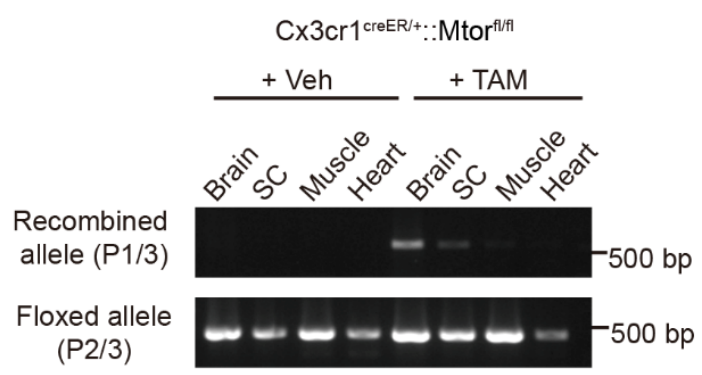

Figure 4-figure supplement 1. Stratagem for generating $M t o r-c K O^{M G}$ mice.

(A) Schematic showing the generation of Mtor-cKO ${ }^{M G}$ mice. Exons 1-5 of the Mtor gene is flanked by loxP sites and excised in microglia expressing Cx3cr1-Cre recombinase after TAM administration. The position of P1, P2 and P3 primers and the size of the DNA segments amplified by primer pairs are illustrated.

(B) Agarose gel electrophoresis of P1, P2 and P3 PCR products showing that Cremediated recombination is specifically occurred in the central nervous system (brain and spinal cord), but not in other peripheral tissues (muscle or heart). TAM, tamoxifen; Veh, vehicle.

Figure 4-figure supplement 1-source data 1. Original pictures of the blots presented in Figure B. 

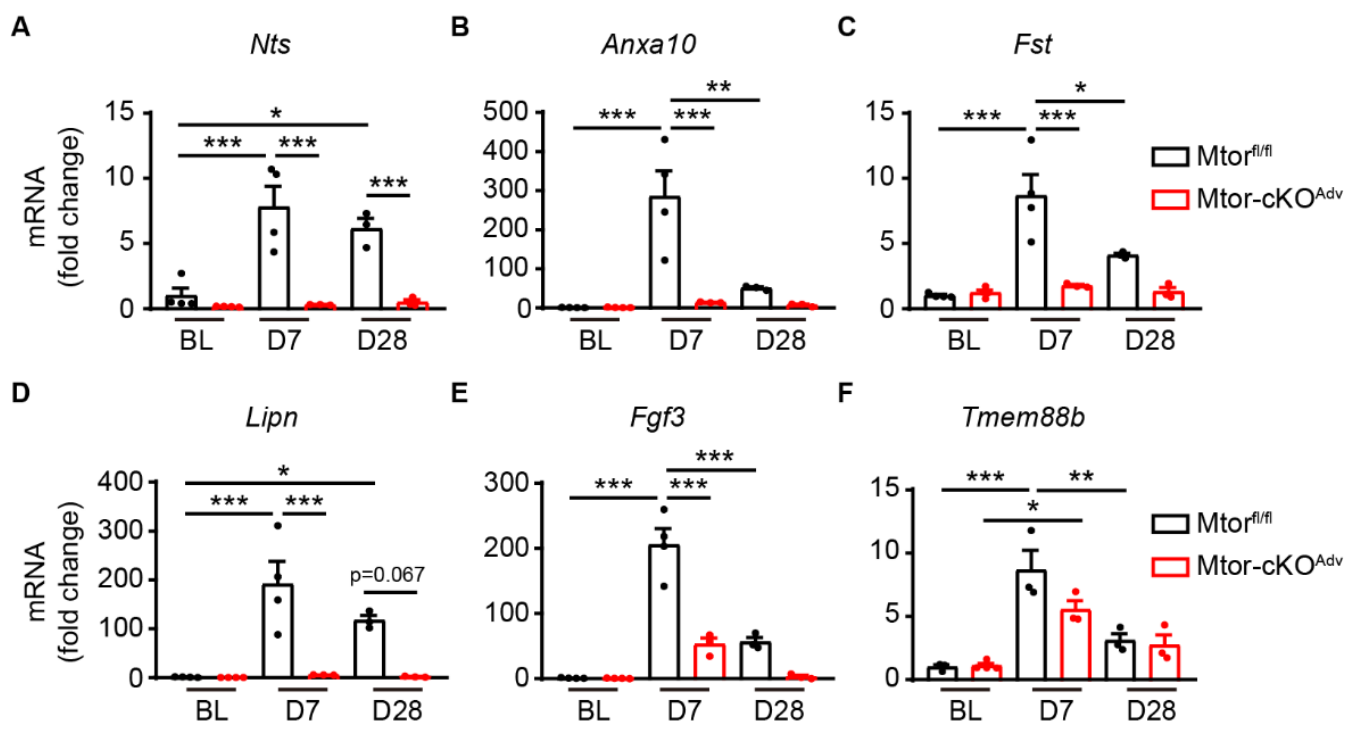

G
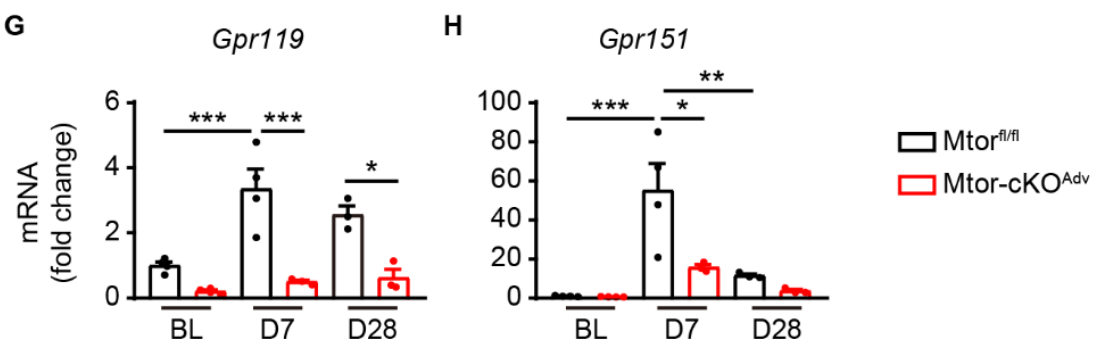

$\square$ Mtor-cKOAdv

Figure 5-figure supplement 1. Quantitative RT-PCR of downregulated DEGs identified in RNA sequencing.

(A) Nts, Neurotensin; (B) Anxa10, Annexin A10; (C) Fst, Follistatin; (D) Lipn, Lipase family member N; (E) Fgf3, Fibroblast growth factor 3; (F) Tmem88b, Transmembrane protein 88b; (G) Gpr119, G protein-coupled receptor 119; (H) Gpr151. n=3-4 mice per time point per group. hoc tests among groups. BL, baseline; D, day; DEGs, differentially expressed genes.

Figure 5-figure supplement 1-source data 1. Source data used to generate Figure AH. 

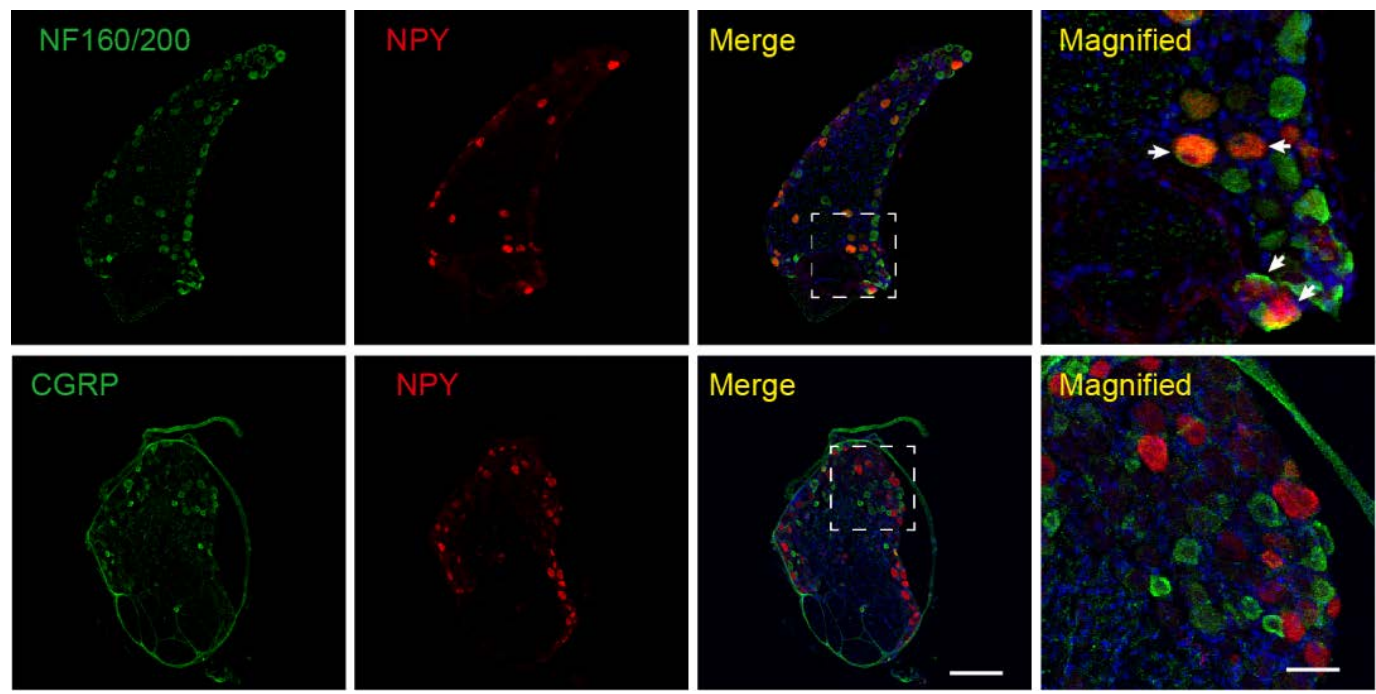

1118 images, respectively.
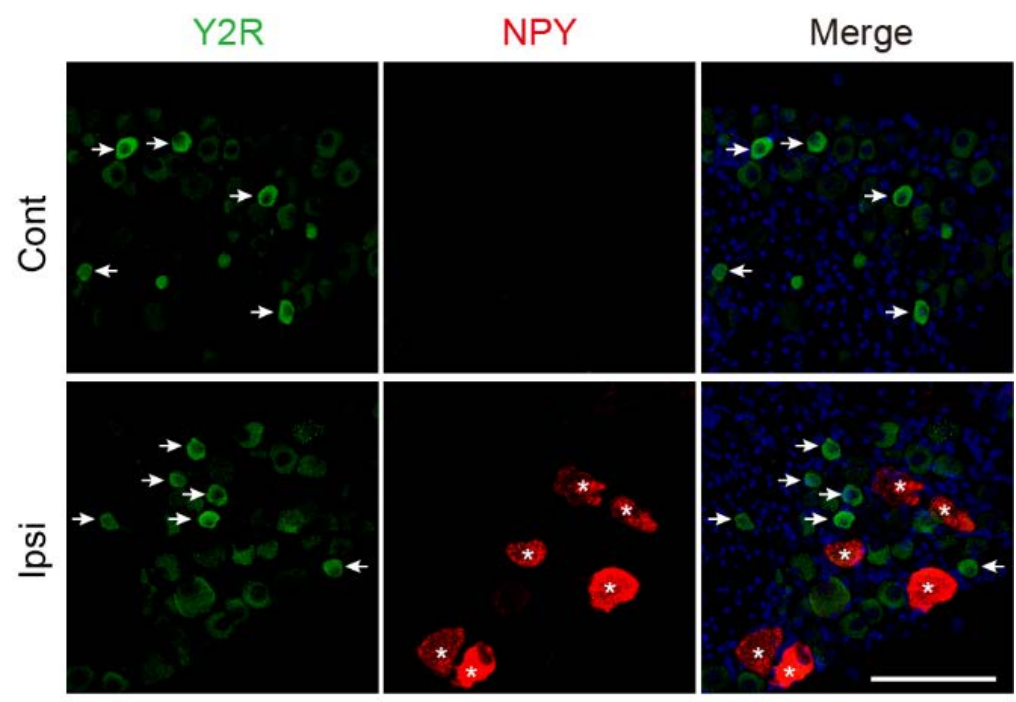

Figure 7-figure supplement 1. Distinct expression pattern of NPY (*) and Y2R 